\title{
Conformational Analysis and Rotational Barriers of Alkyl- and Phenyl-Substituted Urea Derivatives
}

\author{
Vyacheslav S. Bryantsev, Timothy K. Firman, and Benjamin P. Hay* \\ Chemical Sciences Division. Pacific Northwest National Laboratory, P.O. Box 999, \\ Richland, Washington 99354.
}

Supporting Information Available. Cartesian coordinates and energies (Hartrees) for the MP2/aug-cc-pVDZ, B3LYP/DZVP2, and MMFF94+ optimized equilibrium and transition state geometries, and Tables comparing geometric data different levels of theory to that observed in X-ray crystal structures for 3 - 6 . 


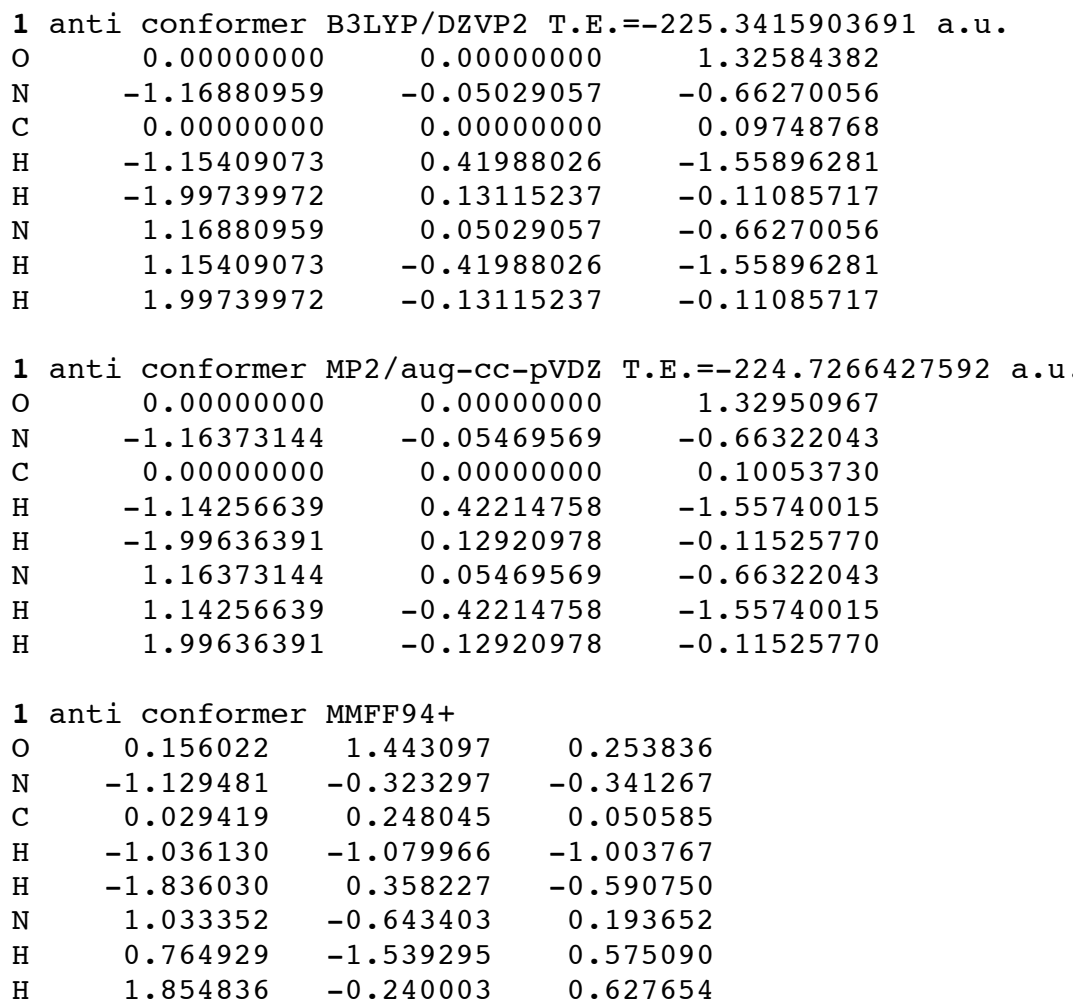

1 syn conformer B3LYP/DZVP2 T.E. $=-225.3399697035 \mathrm{a} \cdot \mathrm{u}$.

$\begin{array}{lrrr}\mathrm{O} & 1.37173294 & 0.06990699 & 0.00000000 \\ \mathrm{~N} & -0.60419079 & 0.05723776 & -1.17314329 \\ \mathrm{C} & 0.14271854 & 0.03075953 & 0.00000000 \\ \mathrm{H} & -1.55498585 & -0.28431800 & -1.17221511 \\ \mathrm{H} & -0.06352087 & -0.15009690 & -2.00137671 \\ \mathrm{~N} & -0.60419079 & 0.05723776 & 1.17314329 \\ \mathrm{H} & -1.55498585 & -0.28431800 & 1.17221511 \\ \mathrm{H} & -0.06352087 & -0.15009690 & 2.00137671\end{array}$

$\begin{array}{lrrr}1 & \text { syn conformer MP2/aug-CC-pVDZ T.E. }=-224.7249535252 \mathrm{a} . \mathrm{u} . \\ \mathrm{O} & 1.37576227 & 0.07225401 & 0.00000000 \\ \mathrm{~N} & -0.60288312 & 0.05582297 & -1.16831776 \\ \mathrm{C} & 0.14628171 & 0.02826183 & 0.00000000 \\ \mathrm{H} & -1.55574733 & -0.28280884 & -1.16182841 \\ \mathrm{H} & -0.06786331 & -0.15011593 & -2.00211529 \\ \mathrm{~N} & -0.60288312 & 0.05582297 & 1.16831776 \\ \mathrm{H} & -1.55574733 & -0.28280884 & 1.16182841 \\ \mathrm{H} & -0.06786331 & -0.15011593 & 2.00211529\end{array}$

1 syn conformer MMFF94+

$\begin{array}{rrrr}\mathrm{O} & 0.162314 & 1.480165 & -0.255870 \\ \mathrm{~N} & 1.071972 & -0.570837 & 0.000692 \\ \mathrm{~N} & -1.150140 & -0.355013 & -0.353248 \\ \mathrm{C} & 0.034885 & 0.268269 & -0.194738 \\ \mathrm{H} & 0.917190 & -1.484439 & 0.394357 \\ \mathrm{H} & 1.947785 & -0.118769 & 0.228933 \\ \mathrm{H} & -1.298695 & -1.268384 & 0.043369 \\ \mathrm{H} & -1.952211 & 0.260379 & -0.392056\end{array}$

1 planar conformer B3LYP/DZVP2 T.E.=-225.3390382031 a.u. (-406.27 cm-1; -326.49 $\mathrm{cm}-1$ )

$\begin{array}{lllr}\mathrm{O} & 0.00000000 & 0.00000000 & 1.37045980 \\ \mathrm{~N} & 1.16688639 & 0.00000000 & -0.60216429 \\ \mathrm{C} & 0.00000000 & 0.00000000 & 0.13866313\end{array}$




$\begin{array}{lrll}\mathrm{H} & 1.19240409 & 0.00000000 & -1.60899982 \\ \mathrm{H} & 2.03122750 & 0.00000000 & -0.08458170 \\ \mathrm{~N} & -1.16688639 & 0.00000000 & -0.60216429 \\ \mathrm{H} & -1.19240409 & 0.00000000 & -1.60899982 \\ \mathrm{H} & -2.03122750 & 0.00000000 & -0.08458170\end{array}$

1 planar conformer MP2/aug-CC-pVDZ T.E.=-224.7241858647 a.u. (-388.90 cm-1; $300.99 \mathrm{~cm}-1)$

$\begin{array}{lrrr}\mathrm{O} & 0.00000000 & 0.00000000 & 1.37442011 \\ \mathrm{~N} & 1.16207741 & 0.00000000 & -0.60064139 \\ \mathrm{C} & 0.00000000 & 0.00000000 & 0.14239415 \\ \mathrm{H} & 1.17886485 & 0.00000000 & -1.60807171 \\ \mathrm{H} & 2.03196319 & 0.00000000 & -0.09087837 \\ \mathrm{~N} & -1.16207741 & 0.00000000 & -0.60064139 \\ \mathrm{H} & -1.17886485 & 0.00000000 & -1.60807171 \\ \mathrm{H} & -2.03196319 & 0.00000000 & -0.09087837\end{array}$

$\begin{array}{lrrr}1 & \text { plane conformer MMFF94+ } & \\ \mathrm{N} & -0.890902 & -0.887285 & 0.000000 \\ \mathrm{C} & 0.197334 & -0.094594 & 0.000000 \\ \mathrm{O} & 1.337290 & -0.530155 & 0.000000 \\ \mathrm{~N} & -0.085053 & 1.221793 & 0.000000 \\ \mathrm{H} & -0.739504 & -1.886423 & 0.000000 \\ \mathrm{H} & -1.831478 & -0.531082 & 0.000000 \\ \mathrm{H} & -1.023510 & 1.583541 & 0.000000 \\ \mathrm{H} & 0.694018 & 1.865412 & 0.000000\end{array}$

\begin{tabular}{lccc}
1 & TS1 & \multicolumn{1}{l}{ Conformer B3LYP/DZVP2 T.E. $=-225.3287710939$} \\
$\mathrm{O}$ & -1.31738904 & -0.37313432 & -0.03982240 \\
$\mathrm{~N}$ & 0.37355663 & 1.30525533 & -0.01572716 \\
$\mathrm{C}$ & -0.13126721 & -0.06267445 & -0.01611018 \\
$\mathrm{H}$ & -0.02718349 & 1.76524008 & -0.83362562 \\
$\mathrm{H}$ & -0.05512490 & 1.77483670 & 0.78234146 \\
$\mathrm{~N}$ & 0.89236106 & -0.96463484 & 0.00727726 \\
$\mathrm{H}$ & 1.84482041 & -0.63843527 & 0.05841804 \\
$\mathrm{H}$ & 0.67698105 & -1.95026800 & 0.03974875
\end{tabular}

$\begin{array}{lrrr}1 & \text { TS1 } & \text { conformer MP2/aug-CC-pVDZ T.E. }=-224.7136450293 \text { a.u. } \\ \text { O } & -1.31373143 & -0.39025178 & -0.04392836 \\ \mathrm{~N} & 0.35998410 & 1.30623203 & -0.01470142 \\ \mathrm{C} & -0.13049123 & -0.06347991 & -0.01397906 \\ \mathrm{H} & -0.05012042 & 1.75512589 & -0.83769746 \\ \mathrm{H} & -0.09733102 & 1.77281605 & 0.77280707 \\ \mathrm{~N} & 0.90447051 & -0.94887812 & 0.02452595 \\ \mathrm{H} & 1.85408304 & -0.61052182 & 0.04674869 \\ \mathrm{H} & 0.70549237 & -1.93921680 & 0.02972650\end{array}$

1 TS1 conformer MMFF94+

$\begin{array}{lrrr}\mathrm{O} & 0.369400 & 0.160800 & -1.502600 \\ \mathrm{~N} & -1.029300 & -0.234400 & 0.227100 \\ \mathrm{C} & 0.133000 & 0.208400 & -0.308200 \\ \mathrm{H} & -1.195100 & -1.176400 & -0.116000 \\ \mathrm{H} & -1.799000 & 0.263900 & -0.209600 \\ \mathrm{~N} & 0.988000 & 0.685000 & 0.616800 \\ \mathrm{H} & 0.602800 & 0.976100 & 1.502600 \\ \mathrm{H} & 1.799000 & 1.176400 & 0.268100\end{array}$

\begin{tabular}{lcrr}
1 & TS2 & \multicolumn{1}{c}{ Conformer B3LYP/DZVP2 T.E. $=-225.3190003146$} \\
$\mathrm{O} . \mathrm{u}$. & $(-391.58 \mathrm{Cm}-1)$ \\
$\mathrm{O}$ & -1.22928280 & 0.65357449 & 0.01372126 \\
$\mathrm{~N}$ & -0.05470076 & -1.37254632 & 0.00099978 \\
$\mathrm{C}$ & -0.15584657 & 0.07483706 & -0.00062018 \\
$\mathrm{H}$ & 0.45307104 & -1.69587887 & -0.82015935 \\
$\mathrm{H}$ & 0.45145261 & -1.68794254 & 0.82629641 \\
$\mathrm{~N}$ & 1.04666067 & 0.75462299 & 0.01145364 \\
$\mathrm{H}$ & 1.92355750 & 0.28162913 & -0.14287700
\end{tabular}


1 TS2 conformer MP2/aug-CC-pVDZ T.E.= -224.7051685395 a.u.

$\begin{array}{lrrr}\mathrm{O} & -1.22094629 & 0.67067075 & 0.02478251 \\ \mathrm{~N} & -0.07580210 & -1.36910377 & -0.00353799 \\ \mathrm{C} & -0.15290723 & 0.07701285 & 0.00209018 \\ \mathrm{H} & 0.44291803 & -1.68798924 & -0.82351240 \\ \mathrm{H} & 0.44588897 & -1.68821215 & 0.81448829 \\ \mathrm{~N} & 1.05704025 & 0.73643891 & 0.01215184 \\ \mathrm{H} & 1.92536275 & 0.24590716 & -0.13821861 \\ \mathrm{H} & 1.04423254 & 1.74459124 & -0.07011854\end{array}$

1 TS2 conformer MMFF94+

$\begin{array}{lrrr}\mathrm{O} & 1.439850 & 0.874800 & 1.272900 \\ \mathrm{~N} & -0.680450 & 0.057400 & 1.082500 \\ \mathrm{C} & 0.501850 & 0.500800 & 0.590600 \\ \mathrm{H} & -0.912450 & -0.874800 & 0.767700 \\ \mathrm{H} & -1.441750 & 0.651600 & 0.776200 \\ \mathrm{~N} & 0.527350 & 0.537900 & -0.761400 \\ \mathrm{H} & 0.041650 & -0.182600 & -1.272900 \\ \mathrm{H} & 1.441750 & 0.773300 & -1.130300\end{array}$

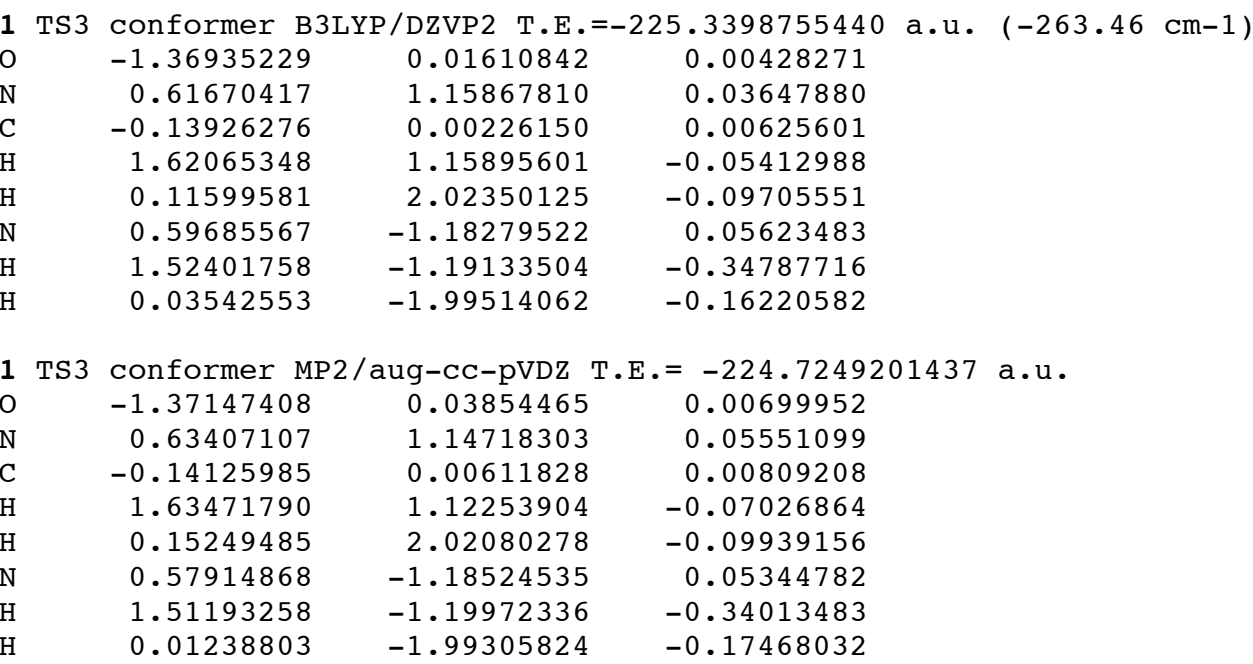

1 TS3 conformer MMFF94+

$\begin{array}{rrrr}\mathrm{O} & 0.164450 & 1.482400 & -0.287500 \\ \mathrm{~N} & 1.069750 & -0.565400 & 0.008200 \\ \mathrm{~N} & -1.152350 & -0.351200 & -0.346500 \\ \mathrm{C} & 0.034650 & 0.272200 & -0.202200 \\ \mathrm{H} & 0.916150 & -1.482400 & 0.394100 \\ \mathrm{H} & 1.950050 & -0.116200 & 0.223600 \\ \mathrm{H} & -1.305350 & -1.244800 & 0.091700 \\ \mathrm{H} & -1.950050 & 0.269400 & -0.394100\end{array}$

\begin{tabular}{lcrr}
2 & Cis & \multicolumn{4}{l}{ Conformer B3LYP/DZVP2 T.E. $=-264.6573023280 \mathrm{a} . \mathrm{u}}$. \\
0 & -1.34252360 & 0.54288240 & 0.13523917 \\
$\mathrm{~N}$ & 0.65196834 & 1.68810486 & -0.07393152 \\
$\mathrm{~N}$ & 0.63329831 & -0.64393941 & 0.04468579 \\
$\mathrm{C}$ & -0.11503100 & 0.52066836 & 0.04764787 \\
$\mathrm{H}$ & 1.56349899 & 1.67671061 & 0.36738362 \\
$\mathrm{H}$ & 0.10996504 & 2.51527951 & 0.14244954 \\
$\mathrm{C}$ & 1.54895882 & -0.58125189 & -0.37998515 \\
$\mathrm{H}$ & -0.04327699 & -1.93479810 & -0.04767387 \\
$\mathrm{H}$ & -0.93705548 & -1.90349742 & 0.57586743 \\
$\mathrm{H}$ & -0.35227038 & -2.17520458 & -1.07288701 \\
& 0.62402242 & -2.71855439 & 0.32135089
\end{tabular}




$\begin{array}{lrrr}2 & \text { cis } & \text { conformer MP2/aug-CC-pVDZ T.E. }=-263.9065414066 \mathrm{a} \cdot \mathrm{u} . \\ \mathrm{O} & -1.33765279 & 0.49658354 & -0.10952009 \\ \mathrm{~N} & 0.64542360 & 1.68673929 & -0.08254252 \\ \mathrm{~N} & 0.65407072 & -0.63358274 & 0.13943955 \\ \mathrm{C} & -0.10932832 & 0.51079761 & -0.01858500 \\ \mathrm{H} & 1.48184792 & 1.70882559 & 0.49091067 \\ \mathrm{H} & 0.06504150 & 2.51192554 & 0.02224122 \\ \mathrm{H} & 1.60349417 & -0.57055999 & -0.20980739 \\ \mathrm{C} & -0.01392294 & -1.92182779 & -0.02519575 \\ \mathrm{H} & -0.87542207 & -1.96269656 & 0.65128577 \\ \mathrm{H} & -0.37467684 & -2.07951839 & -1.05396986 \\ \mathrm{H} & 0.69200891 & -2.71761269 & 0.24416528\end{array}$

\begin{tabular}{lrrr}
\hline & cis conformer & MMFF94+ & \\
0 & -1.177853 & 1.177578 & -0.967438 \\
$\mathrm{~N}$ & -1.506038 & -0.957281 & -0.319456 \\
$\mathrm{~N}$ & 0.491794 & 0.001289 & 0.101977 \\
$\mathrm{C}$ & -0.751053 & 0.164758 & -0.436624 \\
$\mathrm{H}$ & -1.014123 & -1.824919 & -0.477529 \\
$\mathrm{H}$ & -2.376522 & -0.884733 & -0.832525 \\
$\mathrm{H}$ & 0.523417 & -0.667656 & 0.857592 \\
$\mathrm{C}$ & 1.379294 & 1.132801 & 0.198117 \\
$\mathrm{H}$ & 1.435547 & 1.647177 & -0.765269 \\
$\mathrm{H}$ & 0.995672 & 1.821862 & 0.955611 \\
$\mathrm{H}$ & 2.369234 & 0.774299 & 0.489002
\end{tabular}

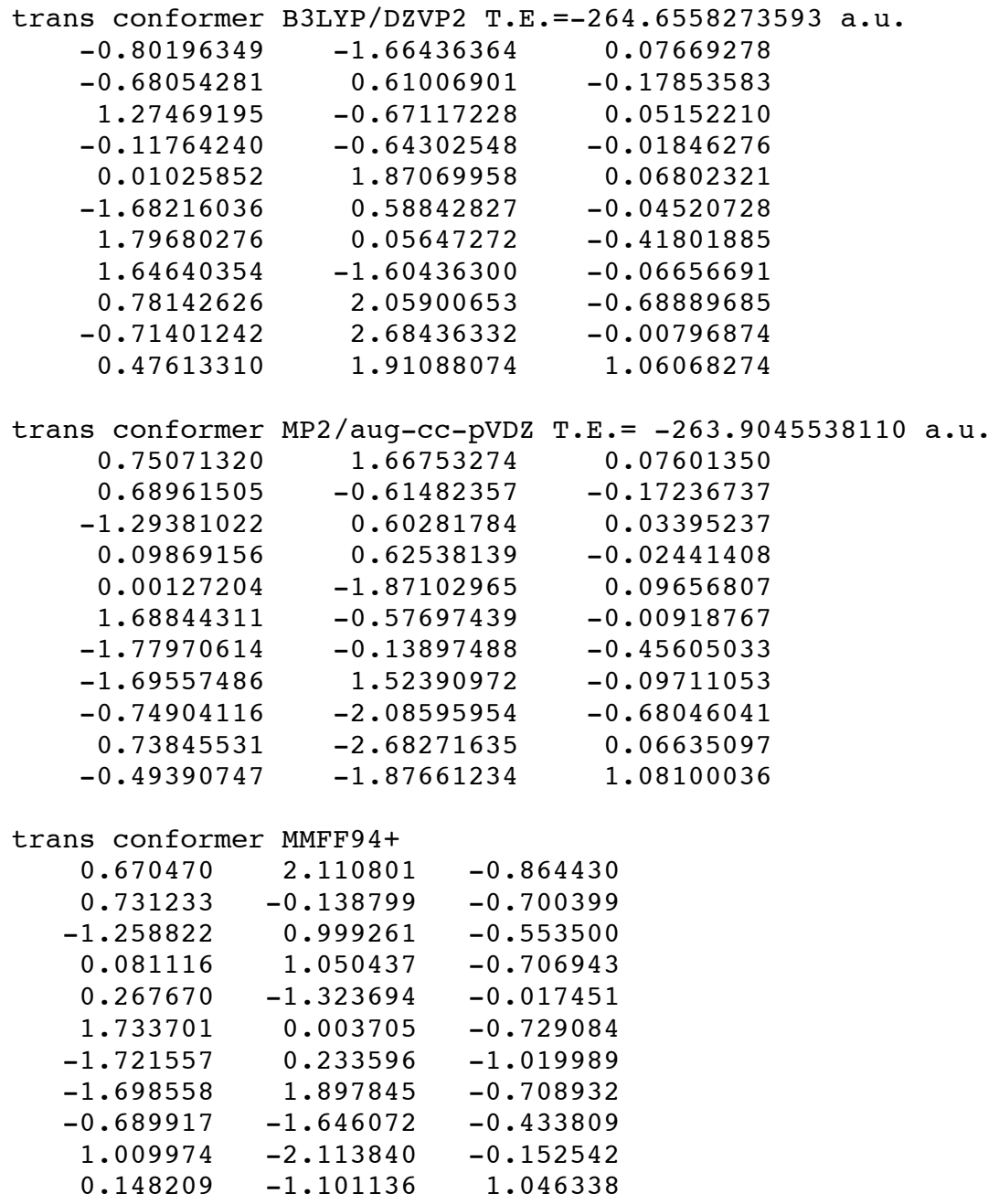




\begin{tabular}{|c|c|c|c|c|c|}
\hline & TS1 & conformer $B 3$ & /DZVP2 T.E.= & 264.6418940622 a.u.(-135.12 & $\mathrm{cm}-1$ \\
\hline ) & & 1.32039905 & -0.77721859 & 0.02813446 & \\
\hline N & & -0.85097291 & -1.46101869 & -0.19915432 & \\
\hline $\mathrm{N}$ & & -0.46192060 & 0.72495675 & 0.50650336 & \\
\hline C & & 0.11718838 & -0.54697424 & 0.10120124 & \\
\hline $\mathrm{H}$ & & -1.82149073 & -1.21057332 & -0.09064948 & \\
\hline $\mathrm{H}$ & & -0.58040589 & -2.39230508 & -0.47996621 & \\
\hline $\mathrm{H}$ & & -0.11960512 & 0.89560541 & 1.45160555 & \\
\hline $\mathrm{C}$ & & 0.01646968 & 1.83240192 & -0.35398547 & \\
\hline $\mathrm{H}$ & & -0.38818530 & 1.70276000 & -1.36191765 & \\
\hline $\mathrm{H}$ & & -0.37069535 & 2.77324132 & 0.04617549 & \\
\hline $\mathrm{H}$ & & 1.11043424 & 1.88590433 & -0.41836353 & \\
\hline 2 & TS1 & conformer MP & aug-cc-pVDz T & $\mathrm{E} .=-263.8915839582 \mathrm{a} \cdot \mathrm{u}$. & \\
\hline $\mathrm{O}$ & & -1.31822690 & 0.74743057 & -0.00053024 & \\
\hline $\mathrm{N}$ & & 0.85197039 & 1.45286880 & -0.19231886 & \\
\hline $\mathrm{N}$ & & 0.47636204 & -0.72672556 & 0.51484772 & \\
\hline C & & -0.11185273 & 0.53454950 & 0.09753231 & \\
\hline $\mathrm{H}$ & & 1.82319468 & 1.20803470 & -0.07362250 & \\
\hline $\mathrm{H}$ & & 0.58033032 & 2.37885850 & -0.49217559 & \\
\hline $\mathrm{H}$ & & 0.11732908 & -0.89469125 & 1.45794463 & \\
\hline C & & -0.01974208 & -1.82108163 & -0.35033583 & \\
\hline $\mathrm{H}$ & & 0.37727406 & -1.67120945 & -1.36417283 & \\
\hline $\mathrm{H}$ & & 0.37055178 & -2.77283171 & 0.03348466 & \\
\hline $\mathrm{H}$ & & -1.11959049 & -1.86477156 & -0.40083978 & \\
\hline 2 & TS1 & conformer MM & $94+$ & & \\
\hline $\mathrm{O}$ & & -1.850050 & 259600 & 1650 & \\
\hline $\mathrm{N}$ & & -1.223850 & 039800 & 6050 & \\
\hline $\mathrm{N}$ & & 0.264250 & 614900 & 50150 & \\
\hline $\mathrm{C}$ & & -1.003750 & 407500 & 79450 & \\
\hline $\mathrm{H}$ & & -0.412050 & 242700 & 59650 & \\
\hline $\mathrm{H}$ & & -2.072650 & 778500 & 8050 & \\
\hline $\mathrm{H}$ & & 0.175250 & 049900 & 59650 & \\
\hline $\mathrm{C}$ & & 1.078850 & 571700 & 6950 & \\
\hline $\mathrm{H}$ & & 1.149550 & 085800 & 55950 & \\
\hline $\mathrm{H}$ & & 0.623250 & 242700 & 31250 & \\
\hline $\mathrm{H}$ & & 2.072650 & 269900 & 35350 & \\
\hline 2 & TS2 & conformer & P/DZVP2 T.E.= & -264.6321811520 a.u. $(-171.47$ & $\mathrm{cm}-1)$ \\
\hline $\mathrm{O}$ & & -1.04070250 & 1.43015649 & 0.05414172 & \\
\hline $\mathrm{N}$ & & 1.18078450 & 0.92273174 & 0.04973530 & \\
\hline $\mathrm{N}$ & & -0.42514405 & -0.74323326 & -0.55821168 & \\
\hline $\mathrm{C}$ & & -0.15430694 & 0.60862665 & -0.11961199 & \\
\hline $\mathrm{H}$ & & 1.88280152 & 0.19924532 & 0.09299720 & \\
\hline $\mathrm{H}$ & & 1.39411223 & 1.81265872 & 0.47941174 & \\
\hline $\mathrm{H}$ & & -0.03437882 & -0.87823128 & -1.48766229 & \\
\hline C & & 0.01554941 & -1.80528826 & 0.35860488 & \\
\hline $\mathrm{H}$ & & -0.46324196 & -1.65872488 & 1.33052274 & \\
\hline $\mathrm{H}$ & & -0.32293005 & -2.76703413 & -0.03655537 & \\
\hline $\mathrm{H}$ & & 1.10583154 & -1.87007092 & 0.51994017 & \\
\hline 2 & TS2 & conformer MP & aug-cc-pVDz T & $\mathrm{E} .=-263.8820732495 \mathrm{a} \cdot \mathrm{u}$. & \\
\hline $\mathrm{O}$ & & 0.99188876 & 1.46098849 & -0.10601972 & \\
\hline $\mathrm{N}$ & & -1.20616696 & 0.86173142 & -0.01611561 & \\
\hline $\mathrm{N}$ & & 0.48281515 & -0.73049916 & 0.53766049 & \\
\hline $\mathrm{C}$ & & 0.14263382 & 0.60687329 & 0.10840923 & \\
\hline $\mathrm{H}$ & & -1.88283635 & 0.11293942 & -0.00147303 & \\
\hline $\mathrm{H}$ & & -1.47850897 & 1.74591543 & -0.42559826 & \\
\hline $\mathrm{H}$ & & 0.12168859 & -0.87508901 & 1.48130646 & \\
\hline $\mathrm{C}$ & & -0.00072717 & -1.78853663 & -0.36317264 & \\
\hline $\mathrm{H}$ & & -1.10168752 & -1.86571750 & -0.45393996 & \\
\hline $\mathrm{H}$ & & 0.41642195 & -1.61157788 & -1.36408033 & \\
\hline $\mathrm{H}$ & & 0.37958860 & -2.75390832 & -0.00370471 & \\
\hline
\end{tabular}




$\begin{array}{lrrr}2 & \text { TS2 } & \text { conformer } & \text { MMFF94+ } \\ \mathrm{O} & -1.094450 & -0.946150 & -1.368550 \\ \mathrm{~N} & -1.486050 & 0.424650 & 0.374150 \\ \mathrm{~N} & 0.547050 & -0.591950 & 0.180450 \\ \mathrm{C} & -0.696150 & -0.404750 & -0.348850 \\ \mathrm{H} & -1.335950 & 0.487450 & 1.368550 \\ \mathrm{H} & -2.450550 & 0.440550 & 0.067050 \\ \mathrm{H} & 0.522750 & -1.163250 & 1.012150 \\ \mathrm{C} & 1.438550 & 0.539350 & 0.289750 \\ \mathrm{H} & 1.404350 & 1.129750 & -0.630250 \\ \mathrm{H} & 1.129750 & 1.163250 & 1.133150 \\ \mathrm{H} & 2.450550 & 0.165750 & 0.461250\end{array}$

3a cis conformer B3LYP/DZVP2 T.E.=-303.9844101686 a.u.

$\begin{array}{rrrr}\mathrm{O} & -1.30755729 & 0.92031734 & 0.39435367 \\ \mathrm{~N} & 0.49267376 & 2.12953035 & -0.39511076 \\ \mathrm{~N} & 0.76114704 & -0.09448129 & 0.26654266 \\ \mathrm{C} & -0.10858561 & 0.97124044 & 0.11799575 \\ \mathrm{H} & 1.45306633 & 2.29887892 & -0.12146424 \\ \mathrm{H} & -0.10525267 & 2.93928099 & -0.28709865 \\ \mathrm{H} & 1.60543845 & -0.06058200 & -0.29051767 \\ \mathrm{C} & 0.24123213 & -1.42696329 & 0.58095400 \\ \mathrm{C} & -0.23615259 & -2.21225574 & -0.64931165 \\ \mathrm{H} & 1.02886603 & -1.97796244 & 1.10636557 \\ \mathrm{H} & -0.58800975 & -1.28504393 & 1.27605490 \\ \mathrm{H} & 0.57919249 & -2.36626590 & -1.36562694 \\ \mathrm{H} & -0.61000302 & -3.19710455 & -0.34866945 \\ \mathrm{H} & -1.04567542 & -1.67614833 & -1.15183219\end{array}$

3a cis conformer MP2/aug-CC-pVDZ T.E.= -303.1006757879 a.u.

$\begin{array}{lrrr}\mathrm{O} & -1.33282523 & -0.82677642 & -0.03984286 \\ \mathrm{~N} & 0.56477847 & -2.06533994 & 0.41306083 \\ \mathrm{~N} & 0.74204184 & 0.10294964 & -0.42630663 \\ \mathrm{C} & -0.10373477 & -0.91758441 & -0.02566850 \\ \mathrm{H} & 1.43743742 & -2.27888307 & -0.05807670 \\ \mathrm{H} & -0.06073326 & -2.86122889 & 0.47867514 \\ \mathrm{H} & 1.67253782 & 0.07770614 & -0.02196069 \\ \mathrm{C} & 0.16739376 & 1.43243765 & -0.63868587 \\ \mathrm{C} & -0.17185303 & 2.15765126 & 0.66452010 \\ \mathrm{H} & 0.88962533 & 2.00983338 & -1.23483298 \\ \mathrm{H} & -0.73758012 & 1.29318439 & -1.24450382 \\ \mathrm{H} & 0.73048678 & 2.30275230 & 1.27873832 \\ \mathrm{H} & -0.60376331 & 3.14776802 & 0.45205926 \\ \mathrm{H} & -0.90364949 & 1.57357765 & 1.23983318\end{array}$

3a cis conformer MMFF94+

$\begin{array}{rrrr}\mathrm{O} & -0.004004 & 1.324822 & 1.801033 \\ \mathrm{~N} & 2.062583 & 1.147689 & 0.919658 \\ \mathrm{~N} & 0.477867 & -0.312312 & 0.251734 \\ \mathrm{C} & 0.769426 & 0.755280 & 1.047846 \\ \mathrm{H} & 2.736460 & 0.399443 & 0.846732 \\ \mathrm{H} & 2.313440 & 1.854359 & 1.600797 \\ \mathrm{H} & 1.077713 & -0.420726 & -0.552869 \\ \mathrm{C} & -0.875287 & -0.816072 & 0.145145 \\ \mathrm{C} & -1.726143 & 0.027357 & -0.788514 \\ \mathrm{H} & -0.808469 & -1.841625 & -0.231159 \\ \mathrm{H} & -1.326907 & -0.843126 & 1.142653 \\ \mathrm{H} & -1.285370 & 0.065714 & -1.790242 \\ \mathrm{H} & -2.732447 & -0.394307 & -0.871857 \\ \mathrm{H} & -1.815759 & 1.056058 & -0.424789\end{array}$

3a trans conformer B3LYP/DZVP2 T.E. $=-303.9817763773$ a.u.

O $\quad-0.65785810 \quad-2.19813681 \quad-0.13544956$

$\mathrm{N} \quad 1.30480627 \quad-1.03326472 \quad 0.17054257$

$\mathrm{N} \quad-0.77436202 \quad 0.06849308 \quad 0.14570677$ 


$\begin{array}{lrrr}\mathrm{C} & -0.08464501 & -1.11884189 & 0.03471167 \\ \mathrm{H} & 1.76412216 & -0.19707581 & -0.16522732 \\ \mathrm{H} & 1.75760275 & -1.89359557 & -0.10975213 \\ \mathrm{H} & -1.77552107 & -0.05940846 & 0.10255067 \\ \mathrm{C} & -0.24308988 & 1.36415850 & 0.56018347 \\ \mathrm{C} & 0.17592609 & 2.27181057 & -0.60922523 \\ \mathrm{H} & -1.01632743 & 1.86750001 & 1.14915631 \\ \mathrm{H} & 0.60034372 & 1.20168450 & 1.23878178 \\ \mathrm{H} & -0.67479309 & 2.47451222 & -1.26685122 \\ \mathrm{H} & 0.55688764 & 3.22762872 & -0.23301756 \\ \mathrm{H} & 0.96056585 & 1.80934883 & -1.21773974\end{array}$

3a trans conformer MP2/aug-CC-pVDZ T.E. $=-303.0972689464$ a.u.

$\begin{array}{lrrr}\mathrm{O} & -0.64228311 & -2.19913568 & -0.12893346 \\ \mathrm{~N} & 1.29443415 & -0.97597908 & 0.14885429 \\ \mathrm{~N} & -0.79814347 & 0.07052104 & 0.15057859 \\ \mathrm{C} & -0.09253934 & -1.10541619 & 0.02881045 \\ \mathrm{H} & 1.71623819 & -0.12654257 & -0.20823686 \\ \mathrm{H} & 1.76893764 & -1.82108274 & -0.14901702 \\ \mathrm{H} & -1.80069090 & -0.06832143 & 0.14890001 \\ \mathrm{C} & -0.25316507 & 1.35653946 & 0.56945088 \\ \mathrm{C} & 0.20493980 & 2.23186521 & -0.60127147 \\ \mathrm{H} & -1.03861061 & 1.87935662 & 1.13512752 \\ \mathrm{H} & 0.57544578 & 1.17887827 & 1.27280184 \\ \mathrm{H} & -0.63744126 & 2.44089697 & -1.27617917 \\ \mathrm{H} & 0.60738380 & 3.18852268 & -0.23373108 \\ \mathrm{H} & 0.98915232 & 1.73471062 & -1.19278405\end{array}$

3a trans conformer MMFF94+

$\begin{array}{lrrr}\mathrm{O} & -2.605841 & -0.180580 & -0.197789 \\ \mathrm{~N} & -0.670612 & -0.364827 & -1.318345 \\ \mathrm{~N} & -0.691896 & -0.301197 & 0.980234 \\ \mathrm{C} & -1.385906 & -0.278193 & -0.178732 \\ \mathrm{H} & 0.217610 & 0.111332 & -1.330651 \\ \mathrm{H} & -1.235170 & -0.211388 & -2.144337 \\ \mathrm{H} & -1.291093 & -0.249931 & 1.794951 \\ \mathrm{C} & 0.707543 & -0.618959 & 1.183813 \\ \mathrm{C} & 1.561010 & 0.635164 & 1.228733 \\ \mathrm{H} & 0.780862 & -1.153909 & 2.136212 \\ \mathrm{H} & 1.055190 & -1.289934 & 0.391915 \\ \mathrm{H} & 1.231847 & 1.302129 & 2.032602 \\ \mathrm{H} & 2.609501 & 0.376761 & 1.405542 \\ \mathrm{H} & 1.499266 & 1.193201 & 0.289120\end{array}$

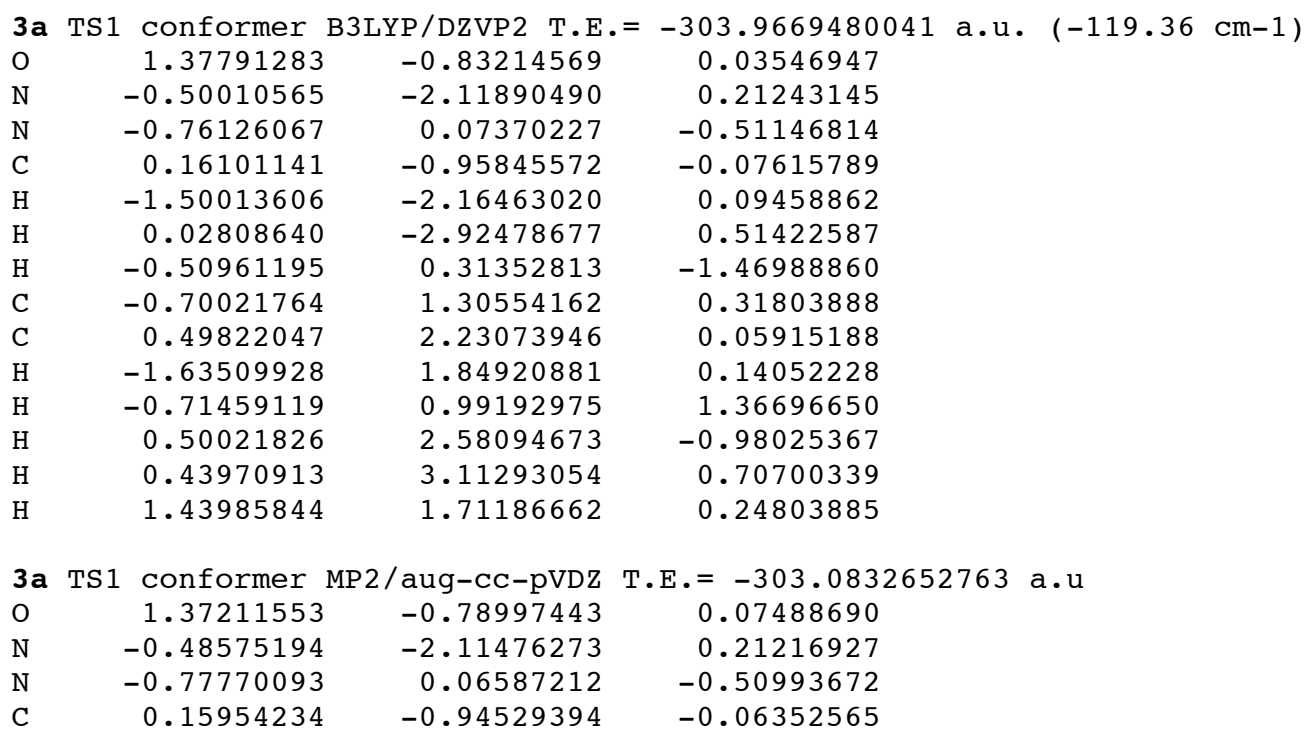




$\begin{array}{rrr}-1.48375751 & -2.17551719 & 0.08023771 \\ 0.04878009 & -2.91173561 & 0.52909004 \\ -0.51127540 & 0.31262639 & -1.46678836 \\ -0.70224315 & 1.29153675 & 0.32613294 \\ 0.49969519 & 2.20079230 & 0.06218468 \\ -1.63815910 & 1.84620467 & 0.15547904 \\ -0.71318499 & 0.96156050 & 1.37693863 \\ 0.50310848 & 2.53321109 & -0.98860722 \\ 0.43983120 & 3.09822355 & 0.69790257 \\ 1.44196127 & 1.67677172 & 0.26495788\end{array}$

a TS1 conformer MMFF94+

$\begin{array}{rrr}-1.225100 & 0.711850 & 1.361100 \\ -2.188900 & 0.529750 & -0.677800 \\ -0.157500 & -0.394650 & -0.343000 \\ -1.186500 & 0.318550 & 0.207100 \\ -1.937000 & 0.520250 & -1.654600 \\ -2.888300 & 1.197950 & -0.385500 \\ -0.075600 & -1.271350 & 0.155800 \\ 1.144900 & 0.247450 & -0.421300 \\ 1.891700 & 0.264650 & 0.903100 \\ 1.725300 & -0.310550 & -1.163000 \\ 1.009500 & 1.271350 & -0.787100 \\ 2.008000 & -0.749050 & 1.300400 \\ 2.888300 & 0.696750 & 0.769300 \\ 1.365400 & 0.860650 & 1.654600\end{array}$

3a TS2 conformer B3LYP/DZVP2 T.E. $=-303.9565272350 \mathrm{a} . \mathrm{u} \cdot(-187.67 \mathrm{~cm}-1)$

$-0.63281354$

$2.13424352-0.16382588$

1.30866218

0.95183974

$-0.12605926$

$-0.74043503$

$-0.07766217$

0.57586436

$-0.05154287$

1.08750429

0.08343432

1.81849270

0.18113923

0.27670164

1.81968484

$-0.51326272$

1.79039188

$-0.36778980$

$-0.25868698$

1.54942829

$-0.73589803$

$-1.29969222$

$-0.24748583$

$-2.24287507$

$-0.11249985$

$-1.64315777$

$-1.85739903$

0.01211313

$-0.85485423$

$-0.98969823$

$-1.29009571$

$-2.52366216$

0.93464726

0.29500660

$-3.16525412$

$-0.67595718$

1.39440077

$-1.79767178$

$-0.50527802$

$3 a$ TS2 conformer MP2/aug-CC-pVDZ T.E. $=-303.0730171345 \mathrm{a} \cdot \mathrm{u}$

\begin{tabular}{|c|c|c|c|c|}
\hline 0 & \multirow{2}{*}{$\begin{array}{r}0.50602114 \\
-1.32968872\end{array}$} & \multicolumn{2}{|c|}{2.15935673} & 0 \\
\hline $\mathrm{N}$ & & \multicolumn{2}{|c|}{0.85046836} & -0 \\
\hline & 0.86143333 & \multicolumn{2}{|c|}{-0.03481742} & -0 \\
\hline C & 0.03342414 & \multicolumn{2}{|c|}{1.08183345} & \\
\hline $\mathrm{H}$ & -1.71153958 & \multicolumn{2}{|c|}{0.08094155} & \\
\hline $\mathrm{H}$ & -1.91857204 & \multicolumn{2}{|c|}{1.66213897} & \\
\hline $\mathrm{H}$ & 0.79021534 & \multicolumn{2}{|c|}{-0.21910839} & \\
\hline & 0.72427462 & \multicolumn{2}{|c|}{-1.26572975} & \\
\hline & -0.42690767 & \multicolumn{2}{|c|}{-2.21587564} & \\
\hline $\mathrm{H}$ & 1.67576238 & \multicolumn{2}{|c|}{-1.81082313} & \\
\hline $\mathrm{H}$ & 0.65346785 & \multicolumn{2}{|c|}{-0.95483572} & \\
\hline & -0.41569834 & \multicolumn{2}{|c|}{-2.45469191} & \\
\hline & -0.31403042 & \multicolumn{2}{|c|}{-3.16264814} & \\
\hline & -1.40961302 & \multicolumn{2}{|c|}{-1.80074933} & \\
\hline & \multicolumn{4}{|c|}{ TS2 conformer MMFF94+ } \\
\hline & -2.603900 & 0.580550 & \multicolumn{2}{|c|}{-0.994500} \\
\hline & -1.571600 & 0.829250 & \multicolumn{2}{|c|}{0.987700} \\
\hline & -0.551600 & -0.358450 & \multicolumn{2}{|c|}{-0.671300} \\
\hline & -1.631000 & & \multicolumn{2}{|c|}{-0.284900} \\
\hline & -1.057600 & $\begin{array}{l}0.374650 \\
0.283750\end{array}$ & 1. & 10 \\
\hline
\end{tabular}




$\begin{array}{rrrr}\mathrm{H} & -2.441400 & 1.229550 & 1.315800 \\ \mathrm{H} & -0.535600 & -1.295150 & -0.294800 \\ \mathrm{C} & 0.746400 & 0.260450 & -0.858800 \\ \mathrm{C} & 1.608000 & 0.227050 & 0.392000 \\ \mathrm{H} & 1.244400 & -0.293350 & -1.661100 \\ \mathrm{H} & 0.604500 & 1.295150 & -1.189700 \\ \mathrm{H} & 1.721700 & -0.796950 & 0.762500 \\ \mathrm{H} & 2.603900 & 0.626150 & 0.175500 \\ \mathrm{H} & 1.174600 & 0.828550 & 1.196100\end{array}$

$\begin{array}{lrrr}\text { 3b cis Conformer MP2/aug-CC-pVDZ } & \text { T.E. }=-303.0997854327 \mathrm{a} \cdot \mathrm{u} \text {. } \\ \mathrm{O} & -1.22679446 & 0.78376531 & -0.56809907 \\ \mathrm{~N} & 0.41779229 & 2.21671290 & 0.20081339 \\ \mathrm{~N} & 0.81956079 & -0.07442452 & 0.04354024 \\ \mathrm{C} & -0.08612877 & 0.95514140 & -0.13357725 \\ \mathrm{H} & 1.06625064 & 2.24273443 & 0.98043374 \\ \mathrm{H} & -0.32001090 & 2.90925173 & 0.26959974 \\ \mathrm{H} & 1.79715298 & 0.18873351 & 0.01503188 \\ \mathrm{C} & 0.46706955 & -1.40605430 & -0.45953276 \\ \mathrm{C} & -0.43766189 & -2.15691364 & 0.51531659 \\ \mathrm{H} & -0.03192899 & -1.30609914 & -1.43630100 \\ \mathrm{H} & 1.40982559 & -1.95295074 & -0.60734117 \\ \mathrm{H} & -1.37080746 & -1.59763304 & 0.66624330 \\ \mathrm{H} & -0.68800976 & -3.15059564 & 0.11283086 \\ \mathrm{H} & 0.06317592 & -2.28663095 & 1.48583118\end{array}$

3b cis conformer MMFF94+

$\begin{array}{lrrr}\mathrm{O} & -1.065822 & 0.416184 & 1.490844 \\ \mathrm{~N} & -2.235128 & 1.054098 & -0.327585 \\ \mathrm{~N} & -0.317707 & -0.092984 & -0.631999 \\ \mathrm{C} & -1.180863 & 0.448001 & 0.276306 \\ \mathrm{H} & -2.009574 & 1.578316 & -1.160721 \\ \mathrm{H} & -2.806154 & 1.568104 & 0.332731 \\ \mathrm{H} & -0.764718 & -0.371395 & -1.493612 \\ \mathrm{C} & 0.803086 & -0.898955 & -0.192282 \\ \mathrm{C} & 1.953404 & -0.050766 & 0.321803 \\ \mathrm{H} & 0.461954 & -1.592260 & 0.584167 \\ \mathrm{H} & 1.135408 & -1.486677 & -1.054181 \\ \mathrm{H} & 1.658485 & 0.536952 & 1.196779 \\ \mathrm{H} & 2.796493 & -0.687027 & 0.607985 \\ \mathrm{H} & 2.296549 & 0.648290 & -0.448146\end{array}$

$\begin{array}{lrrrr}\text { 3b } & \text { trans Conformer } & \text { B 3LYP/DZVP2 T.E. }= & -303.9822578460 & \mathrm{a} . \mathrm{u} . \\ \mathrm{O} & -0.55898278 & -2.01562771 & 0.60959279 \\ \mathrm{~N} & 1.31579237 & -0.98381850 & -0.23253148 \\ \mathrm{~N} & -0.80005724 & 0.05310370 & -0.34435961 \\ \mathrm{C} & -0.05103224 & -1.04433611 & 0.04456643 \\ \mathrm{H} & 1.61434368 & -0.44558176 & -1.03517318 \\ \mathrm{H} & 1.75546763 & -1.89158072 & -0.15213578 \\ \mathrm{H} & -1.74041569 & -0.00666274 & 0.02586880 \\ \mathrm{C} & -0.28167055 & 1.39869747 & -0.59565383 \\ \mathrm{C} & 0.18074176 & 2.15089806 & 0.66281356 \\ \mathrm{H} & 0.53765719 & 1.34139588 & -1.32051420 \\ \mathrm{H} & -1.07851889 & 1.95817155 & -1.09636108 \\ \mathrm{H} & 0.98536552 & 1.60736063 & 1.16534387 \\ \mathrm{H} & 0.54808161 & 3.14841240 & 0.39746209 \\ \mathrm{H} & -0.64680756 & 2.26913539 & 1.36984517\end{array}$

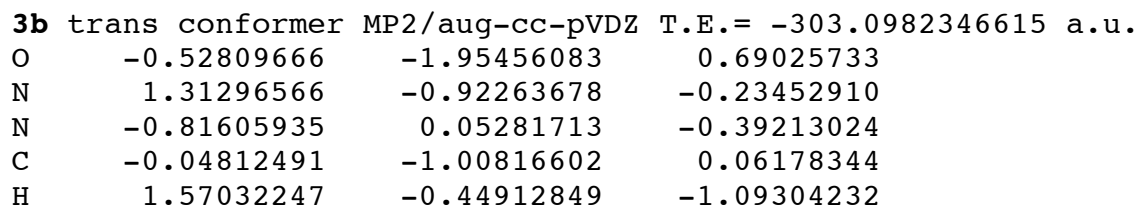




$\begin{array}{lrrr}\mathrm{H} & 1.77631444 & -1.81523968 & -0.10550671 \\ \mathrm{H} & -1.74871636 & 0.00665575 & 0.00738593 \\ \mathrm{C} & -0.28194814 & 1.39730519 & -0.61419124 \\ \mathrm{C} & 0.18354932 & 2.09633936 & 0.66545753 \\ \mathrm{H} & 0.54096843 & 1.34117942 & -1.34328492 \\ \mathrm{H} & -1.07788209 & 1.97786442 & -1.10394869 \\ \mathrm{H} & 0.97670474 & 1.51285648 & 1.15394013 \\ \mathrm{H} & 0.57495203 & 3.09975868 & 0.43704383 \\ \mathrm{H} & -0.65498477 & 2.20452291 & 1.36952860\end{array}$

3b trans conformer MMFF94+

$\begin{array}{lrrr}\mathrm{O} & -0.522328 & 2.118712 & 1.498680 \\ \mathrm{~N} & -1.515924 & 0.303953 & 0.625330 \\ \mathrm{~N} & 0.620595 & 0.880575 & 0.002190 \\ \mathrm{C} & -0.472536 & 1.148981 & 0.754050 \\ \mathrm{H} & -1.700316 & -0.023623 & -0.310691 \\ \mathrm{H} & -2.340663 & 0.636632 & 1.109159 \\ \mathrm{H} & 1.372658 & 1.527822 & 0.205707 \\ \mathrm{C} & 1.000525 & -0.408335 & -0.545861 \\ \mathrm{C} & 1.530820 & -1.346196 & 0.524440 \\ \mathrm{H} & 0.143247 & -0.861229 & -1.052616 \\ \mathrm{H} & 1.775544 & -0.220094 & -1.295705 \\ \mathrm{H} & 0.768233 & -1.558217 & 1.280744 \\ \mathrm{H} & 1.842647 & -2.295546 & 0.078443 \\ \mathrm{H} & 2.393846 & -0.906852 & 1.035697\end{array}$

3b TS1 conformer B3LYP/DZVP2 T.E. = -303.9684627989 a.u. $(-90.69 \mathrm{~cm}-1)$

$\begin{array}{lrrr}\mathrm{O} & -1.03737269 & -1.38995954 & -0.63074579 \\ \mathrm{~N} & 1.13261214 & -1.52375957 & 0.07730865 \\ \mathrm{~N} & -0.20340805 & 0.17905932 & 0.94999271 \\ \mathrm{C} & -0.11360007 & -0.96723697 & 0.05835133 \\ \mathrm{H} & 1.83095090 & -1.15436255 & 0.70357530 \\ \mathrm{H} & 1.31245777 & -2.34857199 & -0.47656570 \\ \mathrm{H} & -0.96913043 & -0.03756169 & 1.58760336 \\ \mathrm{C} & -0.57831526 & 1.42068412 & 0.22006108 \\ \mathrm{C} & 0.58302063 & 1.95046412 & -0.62402158 \\ \mathrm{H} & -1.45906868 & 1.25548063 & -0.41543562 \\ \mathrm{H} & -0.84696411 & 2.15950780 & 0.98180186 \\ \mathrm{H} & 0.86812292 & 1.23478265 & -1.40196617 \\ \mathrm{H} & 0.29198967 & 2.88146544 & -1.12121883 \\ \mathrm{H} & 1.45983192 & 2.14845106 & 0.00069685\end{array}$

$\begin{array}{lrrr}\text { 3b TS1 } & \text { Conformer MP2/aug-CC-pVDZ } & \text { T.E. }=-303.0861342666 \mathrm{a} . \mathrm{u} \\ \mathrm{O} & 1.05215456 & 1.31763312 & -0.64966883 \\ \mathrm{~N} & -1.10773684 & 1.50070257 & 0.08857614 \\ \mathrm{~N} & 0.21323073 & -0.18848091 & 0.98841235 \\ \mathrm{C} & 0.13201926 & 0.93569663 & 0.07068373 \\ \mathrm{H} & -1.78927878 & 1.17539135 & 0.75743672 \\ \mathrm{H} & -1.28389500 & 2.32130518 & -0.47469191 \\ \mathrm{H} & 1.01263385 & 0.02086515 & 1.59254196 \\ \mathrm{C} & 0.54859544 & -1.42731594 & 0.23726861 \\ \mathrm{C} & -0.61192321 & -1.83075186 & -0.66594825 \\ \mathrm{H} & 1.46639900 & -1.29376981 & -0.36209516 \\ \mathrm{H} & 0.73586868 & -2.20998716 & 0.98726501 \\ \mathrm{H} & -0.80164939 & -1.06261596 & -1.43097537 \\ \mathrm{H} & -0.37820418 & -2.77125659 & -1.18646929 \\ \mathrm{H} & -1.52969761 & -1.97235756 & -0.07671328\end{array}$

3b TS1 conformer MMFF94+

$\begin{array}{lrrr}\mathrm{O} & -1.947500 & 0.113650 & 1.047850 \\ \mathrm{~N} & -1.605100 & -0.113450 & -1.180550 \\ \mathrm{~N} & -0.044100 & -0.887150 & 0.258850 \\ \mathrm{C} & -1.252300 & -0.259550 & 0.117250 \\ \mathrm{H} & -0.853800 & -0.101750 & -1.853950 \\ \mathrm{H} & -2.392200 & 0.496850 & -1.350450\end{array}$




$\begin{array}{rrrr}\mathrm{H} & -0.221000 & -1.716550 & 0.811750 \\ \mathrm{C} & 1.009100 & -0.132550 & 0.921650 \\ \mathrm{C} & 1.570100 & 0.971950 & 0.041950 \\ \mathrm{H} & 0.617000 & 0.289150 & 1.853950 \\ \mathrm{H} & 1.802800 & -0.843550 & 1.173650 \\ \mathrm{H} & 0.804500 & 1.716550 & -0.199450 \\ \mathrm{H} & 2.392200 & 1.483450 & 0.551750 \\ \mathrm{H} & 1.949800 & 0.565150 & -0.901150\end{array}$

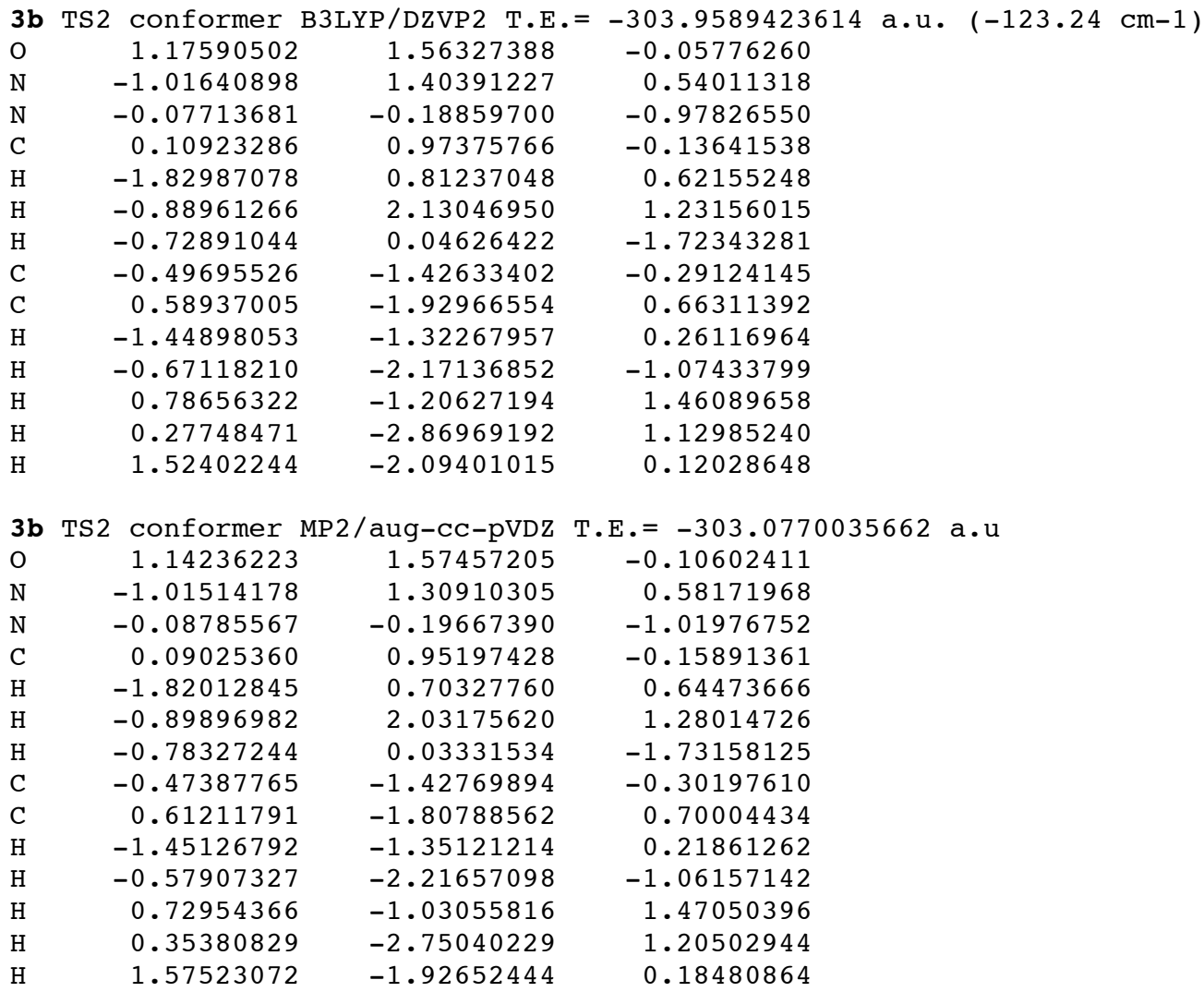

3b TS2 conformer MMFF94+

$\begin{array}{lrrr}\mathrm{O} & -1.552100 & -0.105150 & -1.546400 \\ \mathrm{~N} & -1.666500 & 0.204050 & 0.679500 \\ \mathrm{~N} & 0.151500 & -0.847450 & -0.218300 \\ \mathrm{C} & -1.045800 & -0.232150 & -0.442000 \\ \mathrm{H} & -1.493600 & -0.279650 & 1.546400 \\ \mathrm{H} & -2.617000 & 0.515950 & 0.525000 \\ \mathrm{H} & 0.016500 & -1.758050 & 0.196300 \\ \mathrm{C} & 1.246700 & -0.112050 & 0.391500 \\ \mathrm{C} & 1.762400 & 1.004750 & -0.500000 \\ \mathrm{H} & 0.919100 & 0.292150 & 1.355000 \\ \mathrm{H} & 2.048400 & -0.833450 & 0.580700 \\ \mathrm{H} & 0.989200 & 1.758050 & -0.682200 \\ \mathrm{H} & 2.617000 & 1.501850 & -0.030800 \\ \mathrm{H} & 2.083000 & 0.612950 & -1.471100\end{array}$

3c cis conformer B3LYP/DZVP2 T.E. $=-303.9841859330 \mathrm{a} \cdot \mathrm{u}$.

$\begin{array}{lrrr}\mathrm{O} & 1.27375161 & 1.35521046 & -0.15297531 \\ \mathrm{~N} & -0.90309891 & 2.09579501 & 0.05065222 \\ \mathrm{~N} & -0.42690247 & -0.19093593 & 0.03476963 \\ \mathrm{C} & 0.07592387 & 1.09582160 & -0.03653879 \\ \mathrm{H} & -1.80384813 & 1.88411526 & -0.36103648 \\ \mathrm{H} & -0.53833654 & 3.00133489 & -0.21702608 \\ \mathrm{H} & -1.32858030 & -0.29121464 & 0.48419036\end{array}$




$\begin{array}{lrlr}\mathrm{C} & 0.48965355 & -1.32492967 & 0.17819781 \\ \mathrm{C} & -0.22944710 & -2.64189821 & -0.12061091 \\ \mathrm{H} & 1.31428417 & -1.16375926 & -0.51845040 \\ \mathrm{H} & 0.92548329 & -1.34803969 & 1.18639487 \\ \mathrm{H} & -0.63590538 & -2.64502501 & -1.13672820 \\ \mathrm{H} & 0.46583160 & -3.48124584 & -0.02376058 \\ \mathrm{H} & -1.05547428 & -2.81713956 & 0.57938357\end{array}$

$\begin{array}{lrrr}\text { 3c } & \text { cis } & \text { Conformer MP2/aug-CC-pVDZ } & \text { T.E. }=-303.0994908935 \mathrm{a} . \mathrm{u} . \\ \mathrm{O} & 1.28466159 & -1.32922149 & -0.02122684 \\ \mathrm{~N} & -0.90010455 & -2.08566999 & -0.06288899 \\ \mathrm{~N} & -0.44206560 & 0.19746448 & 0.04250049 \\ \mathrm{C} & 0.07718655 & -1.08584152 & -0.00916537 \\ \mathrm{H} & -1.75242502 & -1.90101807 & 0.45526523 \\ \mathrm{H} & -0.50919573 & -3.00342266 & 0.12104948 \\ \mathrm{H} & -1.36600834 & 0.30516326 & -0.36457907 \\ \mathrm{C} & 0.47215888 & 1.32382321 & -0.15031662 \\ \mathrm{C} & -0.25706771 & 2.63534715 & 0.12008479 \\ \mathrm{H} & 1.30752027 & 1.18689416 & 0.54862637 \\ \mathrm{H} & 0.89725883 & 1.31480749 & -1.16878281 \\ \mathrm{H} & -0.63881362 & 2.66310318 & 1.15099828 \\ \mathrm{H} & 0.42536274 & 3.48528863 & -0.02391980 \\ \mathrm{H} & -1.10598284 & 2.76818556 & -0.56969365\end{array}$

3c cis conformer MMFF94+

$\begin{array}{lrrr}\mathrm{O} & -1.688042 & 1.179402 & -1.295452 \\ \mathrm{~N} & -2.066960 & -0.934747 & -0.607921 \\ \mathrm{~N} & -0.056807 & -0.008095 & -0.176439 \\ \mathrm{C} & -1.288677 & 0.170096 & -0.737591 \\ \mathrm{H} & -1.588402 & -1.814033 & -0.741543 \\ \mathrm{H} & -2.927848 & -0.856966 & -1.136229 \\ \mathrm{H} & -0.074624 & -0.640334 & 0.611127 \\ \mathrm{C} & 0.849803 & 1.117017 & -0.079409 \\ \mathrm{C} & 2.244546 & 0.668503 & 0.310150 \\ \mathrm{H} & 0.874206 & 1.638730 & -1.042535 \\ \mathrm{H} & 0.446881 & 1.809458 & 0.667793 \\ \mathrm{H} & 2.652241 & -0.028140 & -0.429875 \\ \mathrm{H} & 2.917392 & 1.528462 & 0.380794 \\ \mathrm{H} & 2.236266 & 0.158245 & 1.279070\end{array}$

$\begin{array}{lrrr}\text { 3c } & \text { trans COnformer } & \text { B 3LYP/DZVP2 T.E. }=-303.9828890161 \mathrm{a} . \mathrm{u} . \\ \mathrm{O} & -0.99904138 & 2.03568604 & -0.21823822 \\ \mathrm{~N} & 1.21113051 & 1.46023970 & 0.06199792 \\ \mathrm{~N} & -0.47632801 & -0.17349049 & 0.09882649 \\ \mathrm{C} & -0.14912365 & 1.16321533 & -0.02427436 \\ \mathrm{H} & 1.80507268 & 0.84660835 & 0.60362050 \\ \mathrm{H} & 1.37972536 & 2.44758521 & 0.20275524 \\ \mathrm{H} & -1.44164584 & -0.34859811 & -0.14888975 \\ \mathrm{C} & 0.45996573 & -1.28961758 & -0.03480180 \\ \mathrm{C} & -0.29274098 & -2.62107145 & 0.00266861 \\ \mathrm{H} & 1.03533295 & -1.21032471 & -0.96727398 \\ \mathrm{H} & 1.17849400 & -1.26764035 & 0.79554813 \\ \mathrm{H} & -1.00069940 & -2.69569177 & -0.83012945 \\ \mathrm{H} & 0.40908911 & -3.45576642 & -0.08007175 \\ \mathrm{H} & -0.84823623 & -2.73120887 & 0.93933466 \\ & & & \\ \text { 3c } & & & \\ \mathrm{O} & -0.99912741 & 2.03245587 & -0.23092589 \\ \mathrm{~N} & 1.20775684 & 1.42718767 & 0.04439703 \\ \mathrm{~N} & -0.48794539 & -0.17802468 & 0.13558764 \\ \mathrm{C} & -0.15740631 & 1.15519801 & -0.02192833 \\ \mathrm{H} & 1.78884612 & 0.81490063 & 0.60456994 \\ \mathrm{H} & 1.39595659 & 2.41526805 & 0.16760644 \\ \mathrm{H} & -1.45164897 & -0.35958963 & -0.12533787 \\ \mathrm{C} & 0.46216484 & -1.27754129 & -0.01756387\end{array}$




$\begin{array}{lrrr}\mathrm{C} & -0.28620825 & -2.60657181 & -0.00708175 \\ \mathrm{H} & 1.04119381 & -1.16940294 & -0.95102308 \\ \mathrm{H} & 1.17723302 & -1.26195823 & 0.82243888 \\ \mathrm{H} & -0.99135793 & -2.66188794 & -0.85054334 \\ \mathrm{H} & 0.42029045 & -3.44327095 & -0.09944659 \\ \mathrm{H} & -0.84875253 & -2.72683790 & 0.93032303\end{array}$

3c trans conformer MMFF94+

$\begin{array}{lrrr}\mathrm{O} & -2.388704 & 1.207367 & -0.458402 \\ \mathrm{~N} & -1.843967 & -0.944943 & -0.811103 \\ \mathrm{~N} & -0.210108 & 0.622060 & -0.416065 \\ \mathrm{C} & -1.530178 & 0.340362 & -0.546640 \\ \mathrm{H} & -1.219375 & -1.420170 & -1.445516 \\ \mathrm{H} & -2.823132 & -1.062043 & -1.040295 \\ \mathrm{H} & -0.098631 & 1.589013 & -0.134111 \\ \mathrm{C} & 0.777221 & -0.307425 & 0.102619 \\ \mathrm{C} & 2.177903 & 0.265094 & 0.000154 \\ \mathrm{H} & 0.522743 & -0.516839 & 1.147223 \\ \mathrm{H} & 0.719967 & -1.248958 & -0.452273 \\ \mathrm{H} & 2.262732 & 1.198445 & 0.566619 \\ \mathrm{H} & 2.911087 & -0.444170 & 0.395961 \\ \mathrm{H} & 2.436119 & 0.482529 & -1.041570\end{array}$

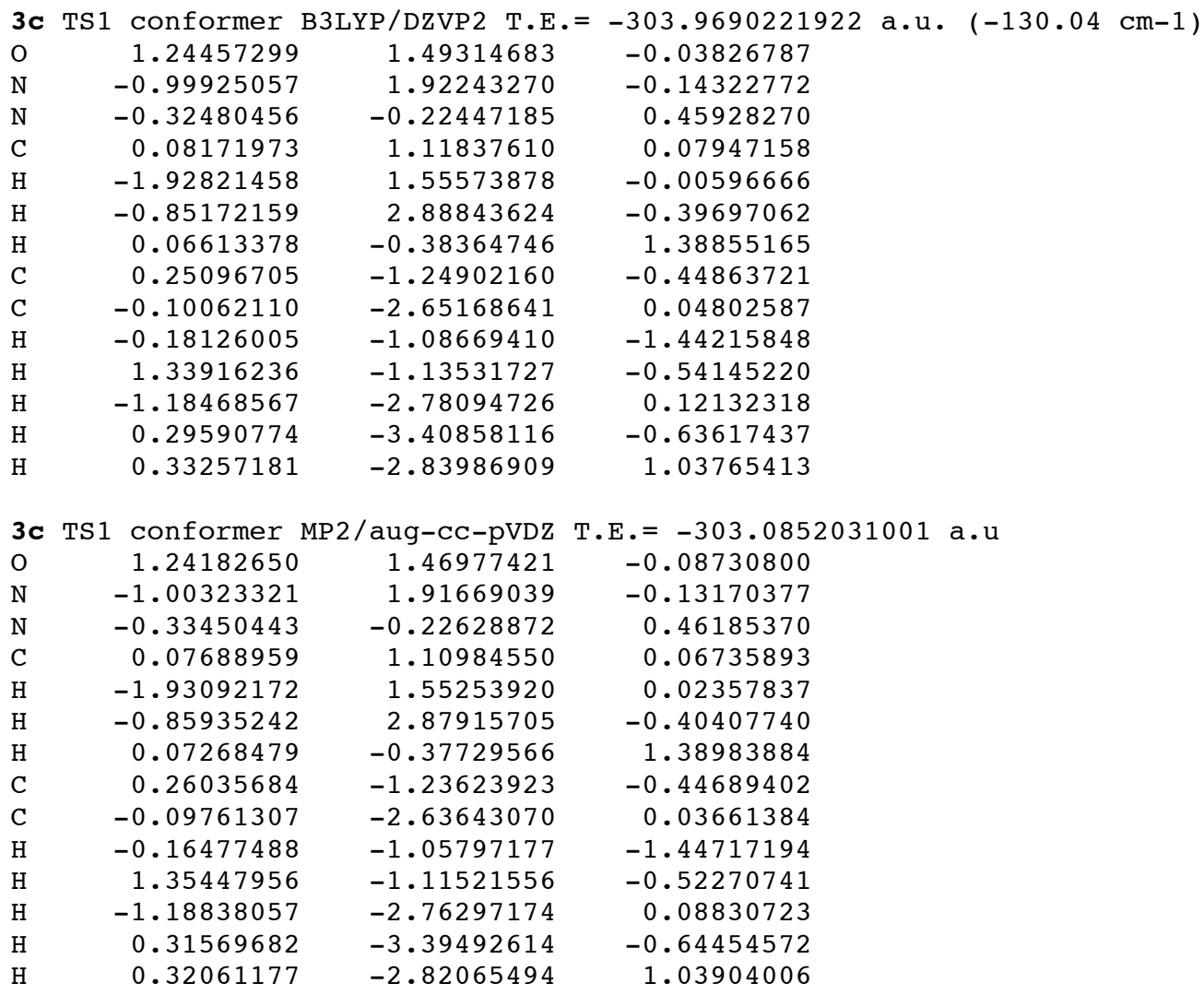

3c TS1 conformer MMFF94+

$\begin{array}{lrrr}\mathrm{O} & -1.156100 & 0.328800 & 2.101450 \\ \mathrm{~N} & -2.206200 & 1.172300 & 0.278750 \\ \mathrm{~N} & -0.543600 & -0.331100 & -0.004250 \\ \mathrm{C} & -1.289200 & 0.388200 & 0.890050 \\ \mathrm{H} & -2.010900 & 1.437500 & -0.674850 \\ \mathrm{H} & -2.657500 & 1.856200 & 0.869950 \\ \mathrm{H} & -0.679200 & -1.307300 & 0.227350 \\ \mathrm{C} & 0.884600 & -0.061400 & 0.006450 \\ \mathrm{C} & 1.584500 & -0.771800 & -1.135150\end{array}$




$\begin{array}{rrrr}\mathrm{H} & 1.034200 & 1.021700 & -0.068650 \\ \mathrm{H} & 1.292100 & -0.388200 & 0.970150 \\ \mathrm{H} & 1.183700 & -0.448300 & -2.101450 \\ \mathrm{H} & 2.657500 & -0.558000 & -1.118550 \\ \mathrm{H} & 1.448800 & -1.856200 & -1.064250\end{array}$

3c TS2 conformer B3LYP/DZVP2 T.E. $=-303.9596324809$ a.u. (-164.91 cm-1)

$\begin{array}{lrrr}\mathrm{O} & 1.07875321 & 1.90616297 & 0.18278268 \\ \mathrm{~N} & -1.16108295 & 1.56800090 & -0.05924341 \\ \mathrm{~N} & 0.36784903 & -0.22693301 & -0.46545198 \\ \mathrm{C} & 0.15615175 & 1.14923517 & -0.07680832 \\ \mathrm{H} & -1.91625982 & 0.89883690 & -0.07497441 \\ \mathrm{H} & -1.34819502 & 2.48011356 & 0.33489347 \\ \mathrm{H} & 0.07140679 & -0.35339090 & -1.43150166 \\ \mathrm{C} & -0.25328396 & -1.24195222 & 0.40458931 \\ \mathrm{C} & 0.18246448 & -2.64427078 & -0.02617871 \\ \mathrm{H} & 0.08485435 & -1.04808700 & 1.42780425 \\ \mathrm{H} & -1.35787690 & -1.19642076 & 0.41858763 \\ \mathrm{H} & 1.27201216 & -2.72900386 & 0.00276199 \\ \mathrm{H} & -0.24984410 & -3.40125048 & 0.63611239 \\ \mathrm{H} & -0.14958633 & -2.86467254 & -1.04760267\end{array}$

3c TS2 conformer MP2/aug-CC-pVDZ T.E.= $-303.0761188338 \mathrm{a} \cdot \mathrm{u}$

$\begin{array}{lrrr}\mathrm{O} & 1.07720676 & 1.91010712 & 0.15989574 \\ \mathrm{~N} & -1.16384327 & 1.53479745 & -0.03095444 \\ \mathrm{~N} & 0.37816097 & -0.22801692 & -0.48668913 \\ \mathrm{C} & 0.15651125 & 1.14338954 & -0.08798734 \\ \mathrm{H} & -1.90961272 & 0.85558071 & -0.06912122 \\ \mathrm{H} & -1.36421198 & 2.44637146 & 0.35981517 \\ \mathrm{H} & 0.04953737 & -0.35008676 & -1.44700066 \\ \mathrm{C} & -0.25952799 & -1.22472453 & 0.39281069 \\ \mathrm{C} & 0.18724177 & -2.62588758 & -0.01096138 \\ \mathrm{H} & 0.06852692 & -1.00326013 & 1.42015785 \\ \mathrm{H} & -1.36810905 & -1.17749756 & 0.38663307 \\ \mathrm{H} & 1.28132900 & -2.70496086 & 0.04651188 \\ \mathrm{H} & -0.26419567 & -3.38081316 & 0.64982475 \\ \mathrm{H} & -0.12281931 & -2.84999236 & -1.04388221\end{array}$

3c TS2 conformer MMFF94+

$\begin{array}{lrrr}\mathrm{O} & -2.409700 & 1.479900 & 0.148550 \\ \mathrm{~N} & -1.200400 & 0.650200 & 1.856450 \\ \mathrm{~N} & -0.722700 & -0.000700 & -0.278650 \\ \mathrm{C} & -1.499600 & 0.766100 & 0.540550 \\ \mathrm{H} & -0.806800 & -0.216200 & 2.187850 \\ \mathrm{H} & -1.872300 & 1.096600 & 2.468150 \\ \mathrm{H} & -0.946000 & -0.980500 & -0.181950 \\ \mathrm{C} & 0.706300 & 0.249400 & -0.326750 \\ \mathrm{C} & 1.333300 & -0.395600 & -1.546950 \\ \mathrm{H} & 0.874300 & 1.332300 & -0.335450 \\ \mathrm{H} & 1.156200 & -0.153300 & 0.587350 \\ \mathrm{H} & 0.890500 & -0.002400 & -2.468150 \\ \mathrm{H} & 2.409700 & -0.201100 & -1.572250 \\ \mathrm{H} & 1.179800 & -1.479900 & -1.539950\end{array}$

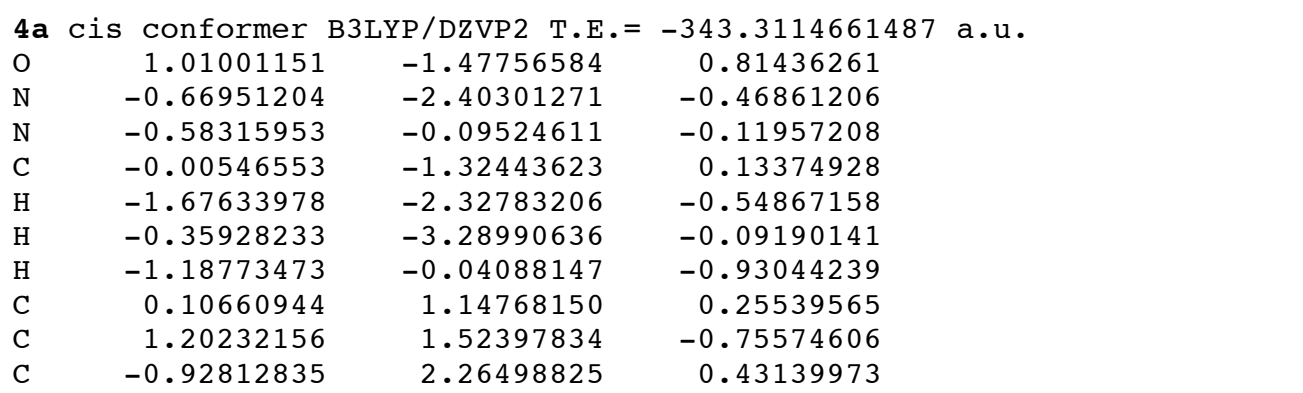




$\begin{array}{rrrr}\mathrm{H} & 0.58323353 & 0.93797942 & 1.21583897 \\ \mathrm{H} & 0.77284469 & 1.72130271 & -1.74561698 \\ \mathrm{H} & 1.73055371 & 2.42735945 & -0.43229138 \\ \mathrm{H} & 1.93115532 & 0.71436839 & -0.84435033 \\ \mathrm{H} & -1.44192565 & 2.48230834 & -0.51372608 \\ \mathrm{H} & -1.68110517 & 1.98849304 & 1.17540658 \\ \mathrm{H} & -0.44035187 & 3.18823918 & 0.75857611\end{array}$

$\begin{array}{lrrr}\text { 4a } & \text { Cis Conformer MP2/aug-CC-pVDZ } & \text { T.E. }=-342.2966435129 \mathrm{a} . \mathrm{u} . \\ \mathrm{O} & -1.09637301 & 1.43034625 & 0.68785083 \\ \mathrm{~N} & 0.62272596 & 2.37587750 & -0.53247524 \\ \mathrm{~N} & 0.62683036 & 0.09211007 & -0.06320514 \\ \mathrm{C} & -0.02986648 & 1.30225960 & 0.08313465 \\ \mathrm{H} & 1.63646404 & 2.33801991 & -0.53517943 \\ \mathrm{H} & 0.25498483 & 3.26796259 & -0.21943855 \\ \mathrm{H} & 1.24452342 & 0.02872185 & -0.86790043 \\ \mathrm{C} & -0.08381676 & -1.14376939 & 0.28538681 \\ \mathrm{C} & -1.16524071 & -1.48302134 & -0.74432059 \\ \mathrm{C} & 0.93981494 & -2.26780886 & 0.43232618 \\ \mathrm{H} & -0.56228067 & -0.94651347 & 1.25573344 \\ \mathrm{H} & -0.70744945 & -1.67653766 & -1.72851864 \\ \mathrm{H} & -1.71995945 & -2.38433721 & -0.44038694 \\ \mathrm{H} & -1.87654769 & -0.65087426 & -0.83739622 \\ \mathrm{H} & 1.45997234 & -2.44580720 & -0.52401081 \\ \mathrm{H} & 1.68983552 & -2.01961794 & 1.19686823 \\ \mathrm{H} & 0.43933512 & -3.20431123 & 0.71932169\end{array}$

$\begin{array}{lcrr}\text { 4a } & \text { Cis Conformer } & \text { MMFF94+ } & \\ \mathrm{O} & -0.624553 & -1.854947 & 1.605985 \\ \mathrm{~N} & -2.318026 & -0.858704 & 0.501660 \\ \mathrm{~N} & -0.239072 & -0.016693 & 0.266591 \\ \mathrm{C} & -1.011730 & -0.976542 & 0.852330 \\ \mathrm{H} & -2.668046 & 0.087391 & 0.456829 \\ \mathrm{H} & -2.910753 & -1.484931 & 1.033481 \\ \mathrm{H} & -0.614394 & 0.335548 & -0.602270 \\ \mathrm{C} & 1.211571 & -0.044060 & 0.392945 \\ \mathrm{C} & 1.823585 & -1.076525 & -0.552393 \\ \mathrm{C} & 1.781510 & 1.343645 & 0.118008 \\ \mathrm{H} & 1.455947 & -0.327266 & 1.424015 \\ \mathrm{H} & 1.580254 & -0.850059 & -1.596490 \\ \mathrm{H} & 2.913737 & -1.099059 & -0.452414 \\ \mathrm{H} & 1.445872 & -2.081800 & -0.337809 \\ \mathrm{H} & 1.550272 & 1.674629 & -0.900655 \\ \mathrm{H} & 1.356175 & 2.080537 & 0.808133 \\ \mathrm{H} & 2.869351 & 1.352037 & 0.241739\end{array}$

4a trans conformer B3LYP/DZVP2 T.E. $=-343.3088011693$ a.u.

$\begin{array}{lrrr}\mathrm{O} & -0.85854700 & -2.39257554 & -0.32596723 \\ \mathrm{~N} & 1.02266929 & -1.58296156 & 0.72361776 \\ \mathrm{~N} & -0.54514582 & -0.12903622 & -0.26093018 \\ \mathrm{C} & -0.16817766 & -1.42490576 & 0.00638758 \\ \mathrm{H} & 1.76250567 & -0.90993871 & 0.57478851 \\ \mathrm{H} & 1.33254091 & -2.54611004 & 0.71810663 \\ \mathrm{H} & -1.43454497 & -0.06972559 & -0.73845403 \\ \mathrm{C} & 0.07708400 & 1.10862336 & 0.21697604 \\ \mathrm{C} & 1.16023596 & 1.62485973 & -0.74981087 \\ \mathrm{C} & -1.01294524 & 2.16376093 & 0.44563709 \\ \mathrm{H} & 0.54162857 & 0.88965911 & 1.18494149 \\ \mathrm{H} & 0.71644662 & 1.89561158 & -1.71365943 \\ \mathrm{H} & 1.65778351 & 2.51004134 & -0.33886109 \\ \mathrm{H} & 1.92405656 & 0.86410493 & -0.94244896 \\ \mathrm{H} & -1.53469673 & 2.39265161 & -0.49144049 \\ \mathrm{H} & -1.74960680 & 1.81426755 & 1.17485814 \\ \mathrm{H} & -0.57403873 & 3.09491638 & 0.81574852\end{array}$




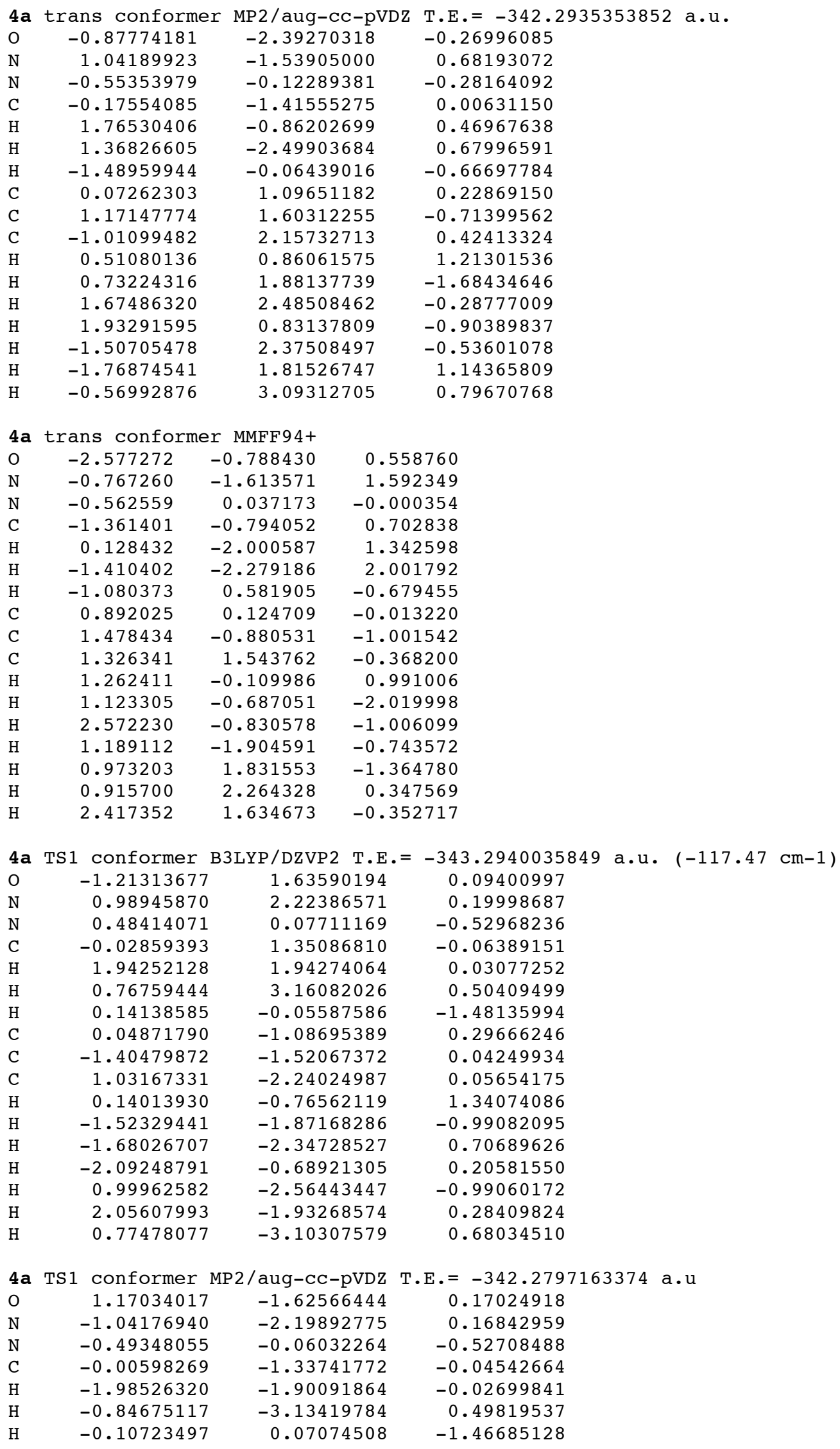




$\begin{array}{lrrr}\mathrm{C} & -0.03318653 & 1.07381466 & 0.32312014 \\ \mathrm{C} & 1.42576899 & 1.47236811 & 0.08016384 \\ \mathrm{C} & -0.97710900 & 2.25156879 & 0.08128216 \\ \mathrm{H} & -0.14718213 & 0.73218334 & 1.36610352 \\ \mathrm{H} & 1.54779924 & 1.81700973 & -0.96115389 \\ \mathrm{H} & 1.71292615 & 2.30172856 & 0.74551694 \\ \mathrm{H} & 2.09948007 & 0.62355519 & 0.25050407 \\ \mathrm{H} & -0.92062554 & 2.57072411 & -0.97237376 \\ \mathrm{H} & -2.01634530 & 1.96996381 & 0.30046070 \\ \mathrm{H} & -0.69668775 & 3.10931853 & 0.71200971\end{array}$

$\begin{array}{lrrr}\text { 4a TS1 conformer MMFF94+ } & \\ \mathrm{O} & -0.969250 & 1.152000 & 1.927950 \\ \mathrm{~N} & -2.244650 & 0.340000 & 0.245150 \\ \mathrm{~N} & -0.089350 & -0.310600 & 0.391450 \\ \mathrm{C} & -1.084550 & 0.455700 & 0.933250 \\ \mathrm{H} & -2.166650 & 0.078200 & -0.726050 \\ \mathrm{H} & -2.957850 & 1.013700 & 0.487450 \\ \mathrm{H} & 0.171650 & -1.009900 & 1.075050 \\ \mathrm{C} & 1.110450 & 0.368800 & -0.096950 \\ \mathrm{C} & 2.056350 & 0.762500 & 1.038750 \\ \mathrm{C} & 1.828650 & -0.532100 & -1.097350 \\ \mathrm{H} & 0.786950 & 1.282400 & -0.611450 \\ \mathrm{H} & 2.363650 & -0.113900 & 1.619950 \\ \mathrm{H} & 2.957850 & 1.243400 & 0.644350 \\ \mathrm{H} & 1.582250 & 1.466800 & 1.729050 \\ \mathrm{H} & 2.155050 & -1.466800 & -0.627850 \\ \mathrm{H} & 1.164350 & -0.795200 & -1.927950 \\ \mathrm{H} & 2.709150 & -0.032300 & -1.514150\end{array}$

$\begin{array}{lrrr}\text { 4a TS2 } & \text { Conformer } & \text { BLYP/DZVP2 T.E. } & -343.2833909246 \mathrm{a} . \mathrm{u} . \\ \mathrm{O} & -1.13097065 & -2.19222496 & 0.09676873 \\ \mathrm{~N} & 1.09747162 & -1.74523848 & 0.08195690 \\ \mathrm{~N} & -0.47348746 & -0.06015142 & -0.58629712 \\ \mathrm{C} & -0.22581442 & -1.40110617 & -0.12762434 \\ \mathrm{H} & 1.84016601 & -1.19445862 & -0.31981206 \\ \mathrm{H} & 1.28591440 & -2.71610589 & 0.29373764 \\ \mathrm{H} & -0.20134406 & 0.06074006 & -1.55822263 \\ \mathrm{C} & -0.06441358 & 1.07805904 & 0.26682884 \\ \mathrm{C} & 1.38323947 & 1.56455696 & 0.05154474 \\ \mathrm{C} & -1.05927666 & 2.22840951 & 0.04754571 \\ \mathrm{H} & -0.16564362 & 0.73198176 & 1.30133675 \\ \mathrm{H} & 1.54253765 & 1.85350463 & -0.99477246 \\ \mathrm{H} & 1.59035095 & 2.44371857 & 0.67080299 \\ \mathrm{H} & 2.12076763 & 0.80188942 & 0.31972491 \\ \mathrm{H} & -1.00640287 & 2.59425315 & -0.98506549 \\ \mathrm{H} & -2.07954400 & 1.88435340 & 0.23204408 \\ \mathrm{H} & -0.83891542 & 3.06985480 & 0.71337919\end{array}$

$\begin{array}{lrrr}\text { 4a TS2 Conformer MP2/aug-CC-pVDZ } & \text { T.E. }=-342.2691785320 \mathrm{a} . \mathrm{u} \\ 0 & -0.99652789 & -2.25483983 & 0.17636055 \\ \mathrm{~N} & 1.19556755 & -1.63549589 & 0.15287028 \\ \mathrm{C} & -0.49580103 & -0.09540903 & -0.55492772 \\ \mathrm{H} & -0.14887423 & -1.40701713 & -0.07365553 \\ \mathrm{H} & 1.88578745 & -1.05773852 & -0.30393994 \\ \mathrm{C} & 1.45466428 & -2.58796993 & 0.38051871 \\ \mathrm{C} & -0.20222281 & 0.02448263 & -1.52532752 \\ \mathrm{H} & -0.08430514 & 1.05325559 & 0.28556449 \\ \mathrm{H} & 1.29592585 & 1.63124924 & -0.05591592 \\ \mathrm{H} & -1.15908302 & 2.13630544 & 0.15930961 \\ \mathrm{H} & -0.07599996 & 0.68278202 & 1.32405877 \\ \mathrm{H} & 1.32600997 & 1.93251607 & -1.11656930 \\ & 1.50141103 & 2.52586364 & 0.55212966 \\ & 2.11086470 & 0.91762189 & 0.13230778 \\ -1.21313530 & 2.49453844 & -0.88170787\end{array}$




$\begin{array}{llll}\mathrm{H} & -2.14087098 & 1.72792002 & 0.43282477 \\ \mathrm{H} & -0.92963880 & 2.99637086 & 0.80768169\end{array}$

\begin{tabular}{lrrr} 
4a TS2 Conformer & \multicolumn{1}{l}{ MMFF94+ } \\
$\mathrm{O}$ & -2.650350 & 0.266200 & -0.003950 \\
$\mathrm{~N}$ & -1.337750 & 1.200600 & 1.562650 \\
$\mathrm{~N}$ & -0.466050 & -0.366400 & 0.148750 \\
$\mathrm{C}$ & -1.550850 & 0.367700 & 0.518950 \\
$\mathrm{H}$ & -0.661450 & 0.922400 & 2.255950 \\
$\mathrm{H}$ & -2.186850 & 1.616900 & 1.923750 \\
$\mathrm{H}$ & -0.272650 & -1.136800 & 0.772250 \\
$\mathrm{C}$ & 0.711350 & 0.262700 & -0.440250 \\
$\mathrm{C}$ & 1.755050 & 0.623200 & 0.616050 \\
$\mathrm{C}$ & 1.315650 & -0.674500 & -1.482150 \\
$\mathrm{H}$ & 0.387050 & 1.184100 & -0.939950 \\
$\mathrm{H}$ & 2.055950 & -0.258500 & 1.192650 \\
$\mathrm{H}$ & 2.650350 & 1.047600 & 0.149350 \\
$\mathrm{H}$ & 1.371450 & 1.364900 & 1.322550 \\
$\mathrm{H}$ & 1.642050 & -1.616900 & -1.028050 \\
$\mathrm{H}$ & 0.579750 & -0.919400 & -2.255950 \\
$\mathrm{H}$ & 2.179550 & -0.212900 & -1.971450
\end{tabular}

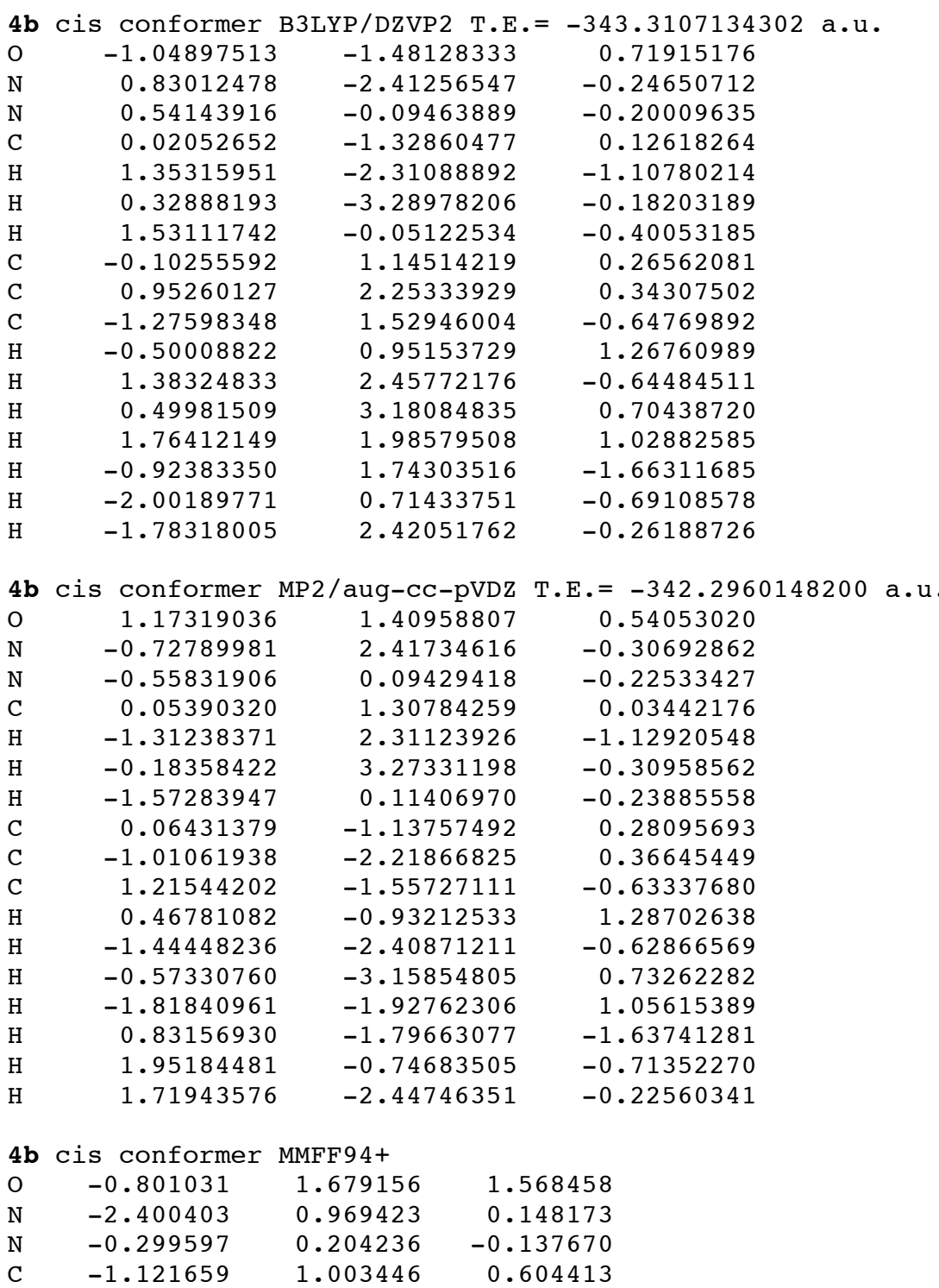




$\begin{array}{lrrr}\mathrm{H} & -2.496161 & 0.965680 & -0.857239 \\ \mathrm{H} & -2.985373 & 1.656648 & 0.608456 \\ \mathrm{H} & -0.786075 & -0.594524 & -0.519779 \\ \mathrm{C} & 1.090271 & -0.002595 & 0.253086 \\ \mathrm{C} & 1.669782 & -1.202612 & -0.493032 \\ \mathrm{C} & 1.935738 & 1.240624 & -0.026671 \\ \mathrm{H} & 1.107531 & -0.211324 & 1.329714 \\ \mathrm{H} & 1.647203 & -1.045796 & -1.577285 \\ \mathrm{H} & 2.706926 & -1.387018 & -0.194368 \\ \mathrm{H} & 1.091345 & -2.108129 & -0.279108 \\ \mathrm{H} & 1.908293 & 1.505357 & -1.089663 \\ \mathrm{H} & 1.574792 & 2.107329 & 0.535873 \\ \mathrm{H} & 2.980209 & 1.073120 & 0.256734\end{array}$

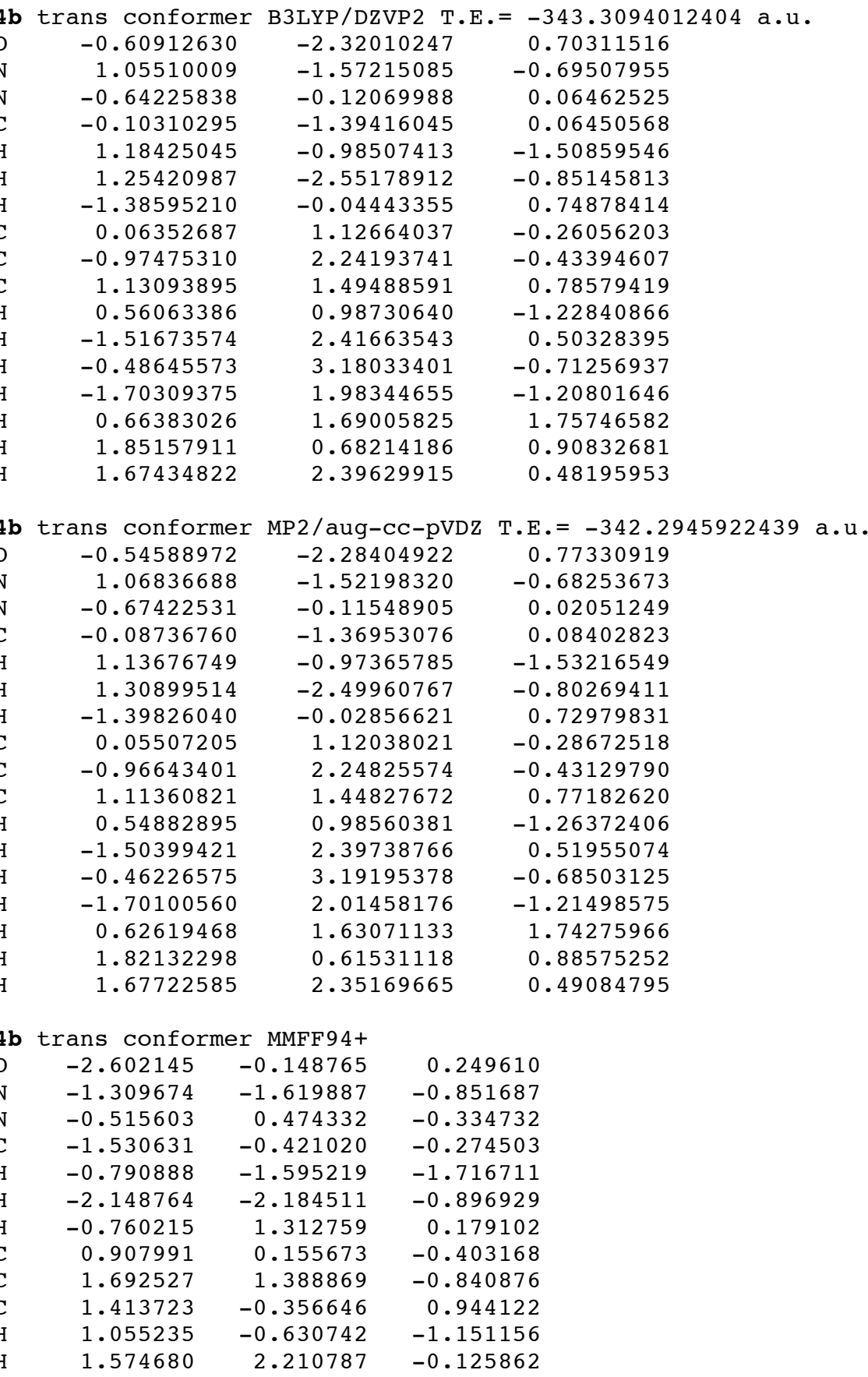




$\begin{array}{rrrr}\mathrm{H} & 2.760242 & 1.162925 & -0.928872 \\ \mathrm{H} & 1.340078 & 1.746131 & -1.814682 \\ \mathrm{H} & 1.267501 & 0.390624 & 1.732047 \\ \mathrm{H} & 0.878688 & -1.262953 & 1.247073 \\ \mathrm{H} & 2.480809 & -0.597075 & 0.894656\end{array}$

4b TS1 Conformer B3LYP/DZVP2 T.E. $=-343.2960441338$ a.u. ( $-86.52 \mathrm{~cm}-1)$

$\begin{array}{lrrr}\mathrm{O} & 0.91165469 & -1.71347035 & 0.89156089 \\ \mathrm{~N} & -0.75467930 & -2.06288557 & -0.63390493 \\ \mathrm{~N} & 0.36764238 & -0.01726374 & -0.68267799 \\ \mathrm{C} & 0.23109923 & -1.31912092 & -0.05119599 \\ \mathrm{H} & -1.23693051 & -1.70081056 & -1.44176922 \\ \mathrm{H} & -0.91702037 & -3.00525708 & -0.30981858 \\ \mathrm{H} & 1.33958827 & 0.03234189 & -0.98886624 \\ \mathrm{C} & 0.15716902 & 1.10632221 & 0.27715602 \\ \mathrm{C} & 0.63440999 & 2.40024376 & -0.38823351 \\ \mathrm{C} & -1.32173920 & 1.18464327 & 0.67124214 \\ \mathrm{H} & 0.75167351 & 0.93284254 & 1.18546048 \\ \mathrm{H} & 0.07151935 & 2.59320961 & -1.30757092 \\ \mathrm{H} & 0.49691395 & 3.25001684 & 0.28767311 \\ \mathrm{H} & 1.69943728 & 2.34761346 & -0.64325302 \\ \mathrm{H} & -1.94576023 & 1.36927129 & -0.20998064 \\ \mathrm{H} & -1.65951391 & 0.25791807 & 1.14548979 \\ \mathrm{H} & -1.47881914 & 1.99910706 & 1.38571986\end{array}$

$\begin{array}{lrrr}\text { 4b TS1 } & \text { Conformer MP2/aug-CC-pVDZ } & \text { T.E. }=-342.2830114212 \\ \mathrm{O} & 0.86203796 & -1.68115992 & 0.92496977 \\ \mathrm{~N} & -0.73590523 & -2.02251239 & -0.67848050 \\ \mathrm{~N} & 0.40927594 & 0.00064209 & -0.69490259 \\ \mathrm{C} & 0.23416687 & -1.29385576 & -0.05854477 \\ \mathrm{H} & -1.17603576 & -1.65635218 & -1.50904437 \\ \mathrm{H} & -0.93769986 & -2.95723531 & -0.35107803 \\ \mathrm{H} & 1.40080532 & 0.03950212 & -0.95095553 \\ \mathrm{C} & 0.17519778 & 1.09792571 & 0.28481723 \\ \mathrm{C} & 0.55540259 & 2.41490737 & -0.38497700 \\ \mathrm{C} & -1.29250814 & 1.08296951 & 0.70442929 \\ \mathrm{H} & 0.80524549 & 0.94422802 & 1.18065122 \\ \mathrm{H} & -0.06753920 & 2.58060789 & -1.27717854 \\ \mathrm{H} & 0.40944640 & 3.25427611 & 0.31149931 \\ \mathrm{H} & 1.61268290 & 2.41200513 & -0.69435379 \\ \mathrm{H} & -1.93864064 & 1.21532669 & -0.17741463 \\ \mathrm{H} & -1.56000275 & 0.13671789 & 1.19820434 \\ \mathrm{H} & -1.49064127 & 1.90003108 & 1.41438218\end{array}$

4b TS1 conformer MMFF94+

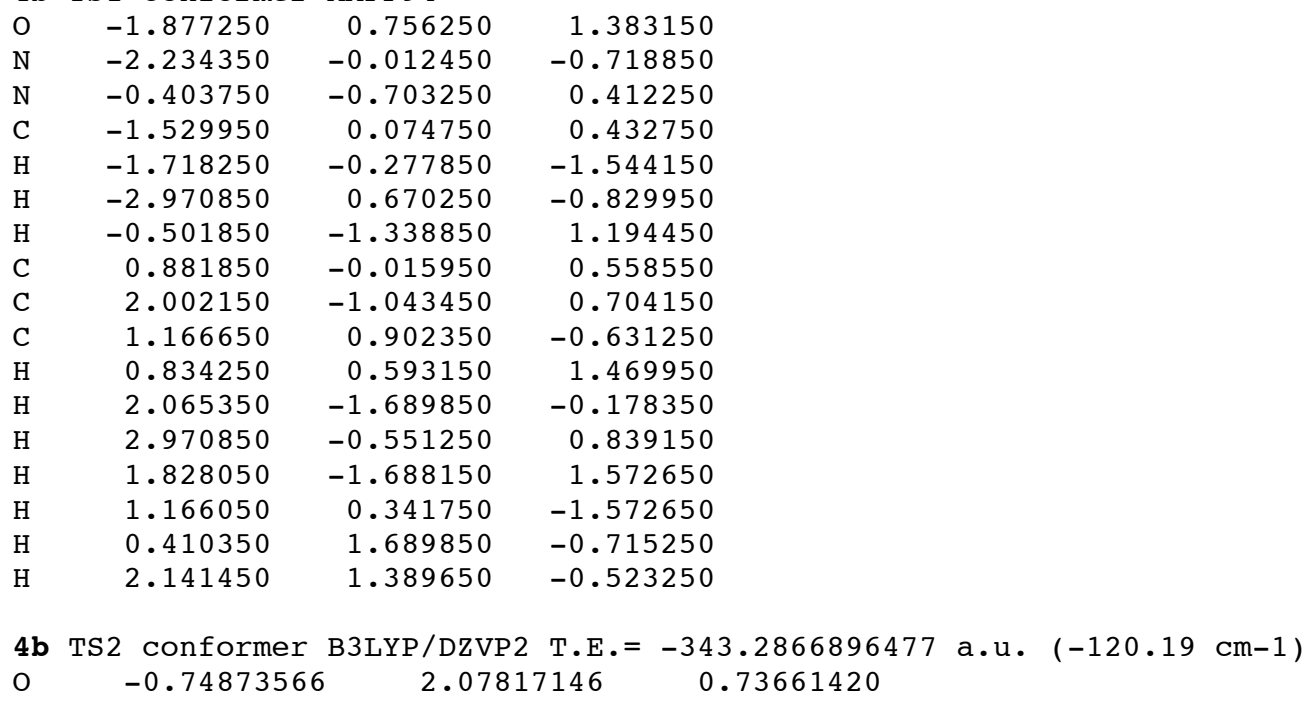




$\begin{array}{lrrr}\mathrm{N} & 0.83906650 & 1.75431477 & -0.86282432 \\ \mathrm{~N} & 0.35365784 & 0.01231842 & 0.70275860 \\ \mathrm{C} & 0.07455599 & 1.34365733 & 0.21265666 \\ \mathrm{H} & 1.37346116 & 1.09021576 & -1.40279693 \\ \mathrm{H} & 0.58416434 & 2.62748972 & -1.30403332 \\ \mathrm{H} & 1.30492766 & -0.01190592 & 1.06488173 \\ \mathrm{C} & 0.13820830 & -1.10513883 & -0.24447321 \\ \mathrm{C} & 0.59322537 & -2.40052935 & 0.43642502 \\ \mathrm{C} & -1.34069086 & -1.16522344 & -0.64464450 \\ \mathrm{H} & 0.73584474 & -0.98607941 & -1.16836242 \\ \mathrm{H} & 0.01809027 & -2.56829434 & 1.35205993 \\ \mathrm{H} & 0.44837195 & -3.25582002 & -0.23089715 \\ \mathrm{H} & 1.65621040 & -2.36020353 & 0.70275555 \\ \mathrm{H} & -1.96386268 & -1.31492925 & 0.24208188 \\ \mathrm{H} & -1.66516451 & -0.23920460 & -1.12961783 \\ \mathrm{H} & -1.51107169 & -1.99071140 & -1.34328602\end{array}$

$\begin{array}{lrrr}\text { 4b TS2 Conformer MP2/aug-CC-pVDZ } & \text { T.E. }=-342.2742013876 \mathrm{a} . \mathrm{u} \\ \mathrm{O} & -0.69640132 & 2.06383798 & 0.78990683 \\ \mathrm{~N} & 0.79629208 & 1.69978105 & -0.89484695 \\ \mathrm{~N} & 0.39866003 & -0.00546118 & 0.72438538 \\ \mathrm{C} & 0.10073958 & 1.32125431 & 0.23252770 \\ \mathrm{H} & 1.32371410 & 1.02646018 & -1.43108257 \\ \mathrm{H} & 0.53368569 & 2.57089903 & -1.33744479 \\ \mathrm{H} & 1.37190243 & -0.02705414 & 1.03732102 \\ \mathrm{C} & 0.15531481 & -1.09324811 & -0.24699997 \\ \mathrm{C} & 0.51326431 & -2.41429866 & 0.42963167 \\ \mathrm{H} & -1.31371471 & -1.05657160 & -0.66358313 \\ \mathrm{H} & 0.78338561 & -0.99388979 & -1.15940269 \\ \mathrm{H} & -0.11662145 & -2.55781618 & 1.31985428 \\ \mathrm{H} & 0.35572808 & -3.25519585 & -0.26241037 \\ \mathrm{H} & 1.56889212 & -2.42591655 & 0.74473608 \\ \mathrm{H} & -1.95150507 & -1.16442263 & 0.22622672 \\ \mathrm{H} & -1.57140556 & -0.10738505 & -1.15718523 \\ & -1.52980399 & -1.87565502 & -1.36593323\end{array}$

4b TS2 conformer MMFF94+

$\begin{array}{lrrr}\mathrm{O} & -2.505700 & -0.012450 & -0.907350 \\ \mathrm{~N} & -1.918200 & 0.807050 & 1.104250 \\ \mathrm{~N} & -0.547000 & -0.688250 & 0.054250 \\ \mathrm{C} & -1.702100 & 0.037950 & 0.011050 \\ \mathrm{H} & -1.532100 & 0.512850 & 1.987150 \\ \mathrm{H} & -2.842100 & 1.219550 & 1.137050 \\ \mathrm{H} & -0.639400 & -1.444450 & 0.717150 \\ \mathrm{C} & 0.748100 & -0.014350 & 0.151050 \\ \mathrm{C} & 1.863900 & -1.053150 & 0.241050 \\ \mathrm{C} & 0.989200 & 0.911050 & -1.042450 \\ \mathrm{H} & 0.749700 & 0.584850 & 1.069250 \\ \mathrm{H} & 1.883500 & -1.693150 & -0.648050 \\ \mathrm{H} & 2.842100 & -0.570850 & 0.338350 \\ \mathrm{H} & 1.720500 & -1.702750 & 1.111450 \\ \mathrm{H} & 0.947100 & 0.357550 & -1.987150 \\ \mathrm{H} & 0.234100 & 1.702750 & -1.089750 \\ \mathrm{H} & 1.970300 & 1.392250 & -0.970950\end{array}$

4c cis conformer B3LYP/DZVP2 T.E. $=-343.3077728088$ a.u.

$\begin{array}{lrrr}\mathrm{O} & -1.01512985 & -0.98513283 & 0.85839701 \\ \mathrm{~N} & 0.38974212 & -2.47392743 & -0.20376315 \\ \mathrm{~N} & 0.57562410 & -0.18792610 & -0.62156356 \\ \mathrm{C} & -0.09309636 & -1.18214862 & 0.06501050 \\ \mathrm{H} & 0.70267655 & -2.64950361 & -1.15102499 \\ \mathrm{H} & -0.24272173 & -3.18328377 & 0.14556275 \\ \mathrm{H} & 1.49085414 & -0.44196433 & -0.96757671 \\ \mathrm{C} & 0.36985943 & 1.25298722 & -0.36340413 \\ \mathrm{C} & -1.05086338 & 1.69417828 & -0.74660423\end{array}$




$\begin{array}{rrrr}\mathrm{C} & 0.73801895 & 1.65586289 & 1.07555105 \\ \mathrm{H} & 1.06811268 & 1.75100559 & -1.04671179 \\ \mathrm{H} & -1.78915071 & 1.22218785 & -0.09596322 \\ \mathrm{H} & -1.13989351 & 2.78112033 & -0.64819350 \\ \mathrm{H} & -1.27301414 & 1.42228663 & -1.78329045 \\ \mathrm{H} & 0.06999017 & 1.16998140 & 1.78975870 \\ \mathrm{H} & 1.76899570 & 1.36980212 & 1.31062942 \\ \mathrm{H} & 0.65032769 & 2.74100748 & 1.19570240\end{array}$

$\begin{array}{lrrr}\text { 4c cis } & \text { Conformer MP2/aug-CC-PVDZ } & \text { T.E. }=-342.2936869421 \mathrm{a} . \mathrm{u} . \\ \mathrm{O} & 0.98782506 & 0.93763538 & 0.87782818 \\ \mathrm{~N} & -0.37036293 & 2.45482817 & -0.21063642 \\ \mathrm{~N} & -0.54414331 & 0.18426230 & -0.68518770 \\ \mathrm{C} & 0.09909389 & 1.16051339 & 0.05266129 \\ \mathrm{H} & -0.64547845 & 2.64113873 & -1.16926070 \\ \mathrm{H} & 0.25379671 & 3.15916521 & 0.16828223 \\ \mathrm{H} & -1.46429605 & 0.44782363 & -1.02075758 \\ \mathrm{C} & -0.36674198 & -1.24886603 & -0.38679861 \\ \mathrm{C} & 1.06525254 & -1.69980850 & -0.68552907 \\ \mathrm{C} & -0.80159474 & -1.60874686 & 1.03870268 \\ \mathrm{H} & -1.03809918 & -1.75811788 & -1.09834046 \\ \mathrm{H} & 1.77120305 & -1.22286972 & 0.00625050 \\ \mathrm{H} & 1.13775740 & -2.79218459 & -0.57090581 \\ \mathrm{H} & 1.34367025 & -1.43873267 & -1.71691589 \\ \mathrm{H} & -0.14948395 & -1.11415885 & 1.77104178 \\ \mathrm{H} & -1.84124950 & -1.29561489 & 1.21988218 \\ \mathrm{H} & -0.74014435 & -2.69809693 & 1.18642762\end{array}$

4c $\mathrm{Cis}$ conformer MMFF94+

$\begin{array}{lrrr}\mathrm{O} & -0.823805 & 0.733384 & 1.287216 \\ \mathrm{~N} & -2.307501 & 0.236049 & -0.329192 \\ \mathrm{~N} & -0.113606 & 0.023447 & -0.795291 \\ \mathrm{C} & -1.040769 & 0.360711 & 0.146071 \\ \mathrm{H} & -2.444284 & 0.552730 & -1.278166 \\ \mathrm{H} & -2.991599 & 0.603791 & 0.321372 \\ \mathrm{H} & -0.463508 & -0.612945 & -1.496717 \\ \mathrm{C} & 1.318511 & -0.037410 & -0.527155 \\ \mathrm{C} & 1.898154 & 1.321005 & -0.138536 \\ \mathrm{C} & 1.668325 & -1.111971 & 0.499661 \\ \mathrm{H} & 1.771474 & -0.327362 & -1.483321 \\ \mathrm{H} & 1.528947 & 1.662980 & 0.833302 \\ \mathrm{H} & 2.990627 & 1.270844 & -0.079671 \\ \mathrm{H} & 1.634289 & 2.081925 & -0.880995 \\ \mathrm{H} & 1.268007 & -0.875365 & 1.490711 \\ \mathrm{H} & 1.258446 & -2.082524 & 0.199722 \\ \mathrm{H} & 2.754205 & -1.217935 & 0.593852\end{array}$

$\begin{array}{lrrr}\text { 4c trans conformer } & \text { B } 3 L Y P / D Z V P 2 & T . E .= & -343.3047727266 \mathrm{a} . \mathrm{u} . \\ \mathrm{O} & 0.06650920 & -2.41254818 & -0.63493323 \\ \mathrm{~N} & -0.89620956 & -1.11296249 & 0.99623514 \\ \mathrm{~N} & 0.72537800 & -0.22692799 & -0.48746069 \\ \mathrm{C} & -0.02712060 & -1.31622080 & -0.07640293 \\ \mathrm{H} & -0.61449578 & -0.44657599 & 1.70193432 \\ \mathrm{H} & -1.24005878 & -1.99033584 & 1.36564379 \\ \mathrm{H} & 1.23186315 & -0.47320150 & -1.32824871 \\ \mathrm{C} & 0.41626466 & 1.21317246 & -0.34955119 \\ \mathrm{C} & -0.96623789 & 1.59158877 & -0.91114982 \\ \mathrm{C} & 0.62899847 & 1.74955222 & 1.07792103 \\ \mathrm{H} & 1.17523802 & 1.69740826 & -0.97365434 \\ \mathrm{H} & -1.76523401 & 1.10444662 & -0.34610884 \\ \mathrm{H} & -1.11496730 & 2.67539059 & -0.85568058 \\ \mathrm{H} & -1.05494783 & 1.28655735 & -1.95836424 \\ \mathrm{H} & -0.18883277 & 1.47927914 & 1.75470416 \\ \mathrm{H} & 1.57071882 & 1.38345197 & 1.49636554 \\ \mathrm{H} & 0.66220391 & 2.84344788 & 1.05766201\end{array}$




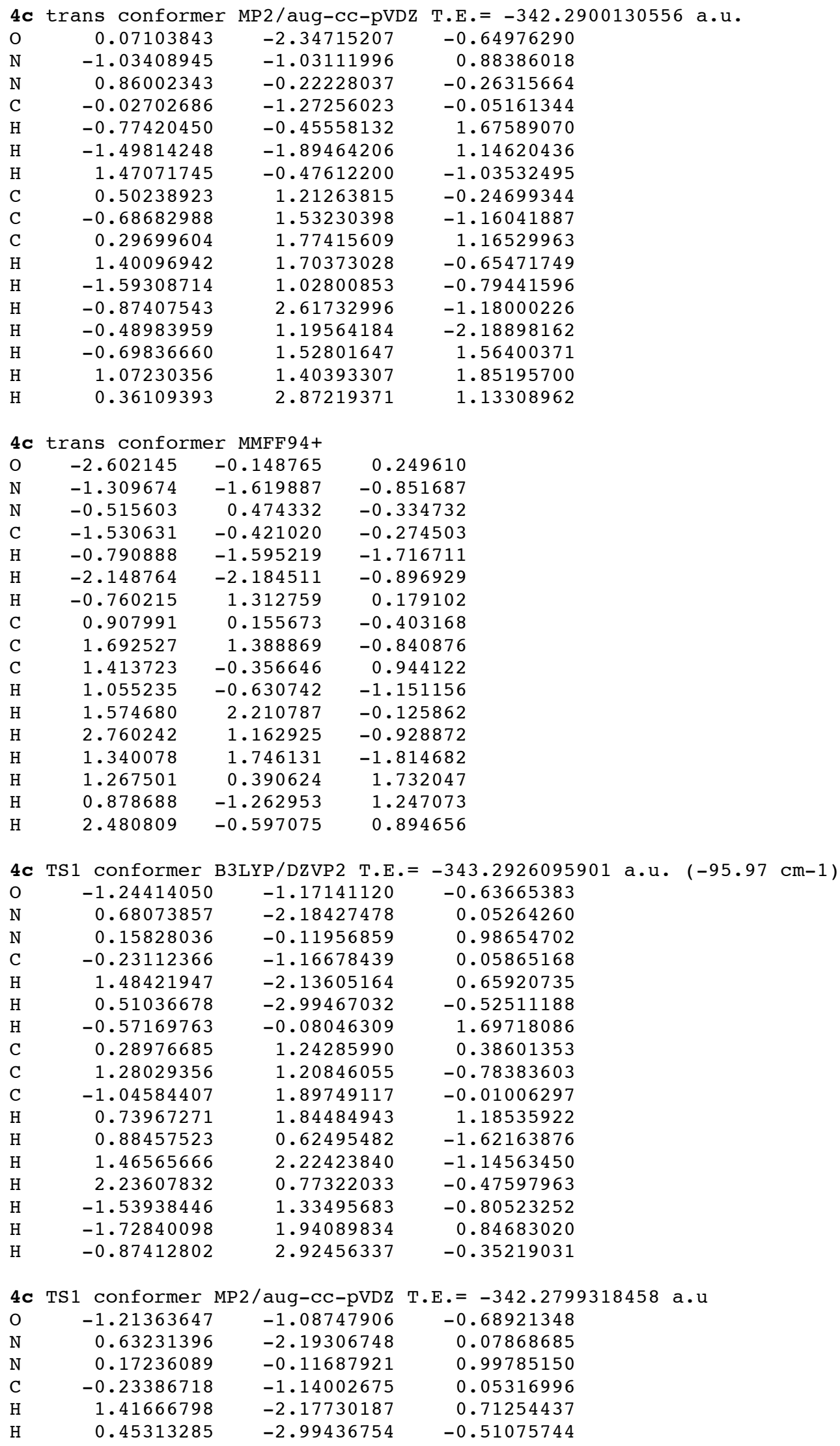




$\begin{array}{rrrr}\mathrm{H} & -0.56655608 & -0.06651750 & 1.70438782 \\ \mathrm{C} & 0.31512478 & 1.23177332 & 0.37777417 \\ \mathrm{C} & 1.27274420 & 1.13765846 & -0.80988219 \\ \mathrm{C} & -1.00801756 & 1.89974186 & -0.01493613 \\ \mathrm{H} & 0.79571090 & 1.84292020 & 1.16039876 \\ \mathrm{H} & 0.82478430 & 0.55010832 & -1.62650444 \\ \mathrm{H} & 1.48766683 & 2.14428182 & -1.19776981 \\ \mathrm{H} & 2.22220867 & 0.66872850 & -0.51294105 \\ \mathrm{H} & -1.50067191 & 1.34678240 & -0.82549627 \\ \mathrm{H} & -1.69548475 & 1.93856784 & 0.84516108 \\ \mathrm{H} & -0.81955985 & 2.93444812 & -0.34333362\end{array}$

4c TS1 conformer MMFF94+

$\begin{array}{lrrr}\mathrm{O} & -1.119850 & 0.788950 & 1.730850 \\ \mathrm{~N} & -2.272850 & 0.189850 & -0.120050 \\ \mathrm{~N} & -0.300450 & -0.818350 & 0.308850 \\ \mathrm{C} & -1.213350 & 0.113650 & 0.719550 \\ \mathrm{H} & -2.107550 & -0.086550 & -1.076150 \\ \mathrm{H} & -2.886050 & 0.978250 & 0.033650 \\ \mathrm{H} & -0.267250 & -1.540250 & 1.017550 \\ \mathrm{C} & 1.067950 & -0.403250 & -0.008850 \\ \mathrm{C} & 1.103850 & 0.592750 & -1.166950 \\ \mathrm{C} & 1.829550 & 0.135550 & 1.201650 \\ \mathrm{H} & 1.569450 & -1.321650 & -0.338150 \\ \mathrm{H} & 0.623750 & 1.540250 & -0.899150 \\ \mathrm{H} & 2.136050 & 0.809050 & -1.461350 \\ \mathrm{H} & 0.579750 & 0.190550 & -2.040850 \\ \mathrm{H} & 1.430450 & 1.096650 & 1.540950 \\ \mathrm{H} & 1.774950 & -0.566050 & 2.040850 \\ \mathrm{H} & 2.886050 & 0.285550 & 0.954750\end{array}$

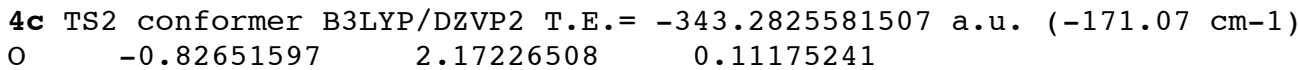

1.29736049

0.11234513

$-0.17422574$

$-0.00510736$

1.90668248

1.32201375

0.46393152

$-0.24700624$

$-1.29957918$

1.09068484

$-0.61826550$

$-0.99374048$

$-1.43506915$

$-2.25815121$

1.44685268

1.86714313

0.96363197
1.26718009

0.67925501

2.15900442

0.15212234

$-1.24487642$

$-1.26959043$

$-1.86035257$

$-1.87168904$

$-0.64653526$

$-2.29116712$

$-0.89685198$

$-1.40623021$

$-1.75428896$

$-2.93166755$
$-0.63380185$

0.99658602

0.15025942

$-0.44236050$

$-1.12856929$

1.78631169

0.40646509

$-0.70870482$

$-0.06087089$

1.22678228

$-1.55586370$

$-1.07703294$

$-0.33904729$

$-0.99141448$

0.70659698

$-0.25151258$

TS2 conformer MP2/aug-CC-pVDZ T.E.= -342.2699540845 a.u

0.67501649

$-2.23707500$

0.09478557

$-1.21588725$

0.25689184

$-0.05942287$

$-1.94011760$

$-1.50169634$

$-0.32270312$

0.33695795

1.27803007

$-0.99568547$

0.81354540

0.84322291

1.44153891

$-1.16019410$

$-0.57911774$

$-0.13172532$

1.00997183

$-1.25957104$

$-0.51595563$

$-2.00495577$

0.17267168

$-0.29597088$

$-0.13655881$

$-1.06031793$

1.21023364

1.14204535

1.84894648

0.39258450

1.88359924

$-0.81059673$

1.83349421

0.51818338

0.02075942

1.16808675

2.14988053

$-1.60732800$

$-1.22065661$

2.24637033

0.71281389

$-0.51892528$ 


$\begin{array}{lllr}\mathrm{H} & -1.42921499 & 1.45123007 & -0.89340197 \\ \mathrm{H} & -1.72562429 & 1.80407984 & 0.84315979 \\ \mathrm{H} & -0.82868602 & 2.95514909 & -0.17122424\end{array}$

4c TS2 conformer MMFF94+

$\begin{array}{lrrr}\mathrm{O} & -2.622600 & 0.303300 & -0.229700 \\ \mathrm{~N} & -1.380400 & 0.838900 & 1.561800 \\ \mathrm{~N} & -0.633200 & -0.755200 & 0.104300 \\ \mathrm{C} & -1.596000 & 0.154500 & 0.416600 \\ \mathrm{H} & -0.843000 & 0.394500 & 2.289000 \\ \mathrm{H} & -2.180500 & 1.377100 & 1.869400 \\ \mathrm{H} & -0.678700 & -1.575700 & 0.690900 \\ \mathrm{C} & 0.725900 & -0.382400 & -0.285200 \\ \mathrm{C} & 0.733600 & 0.613300 & -1.444000 \\ \mathrm{C} & 1.573800 & 0.123600 & 0.879400 \\ \mathrm{H} & 1.177400 & -1.316100 & -0.642600 \\ \mathrm{H} & 0.300200 & 1.575700 & -1.151100 \\ \mathrm{H} & 1.755500 & 0.795700 & -1.792600 \\ \mathrm{H} & 0.151100 & 0.230200 & -2.289000 \\ \mathrm{H} & 1.246400 & 1.108200 & 1.227600 \\ \mathrm{H} & 1.526600 & -0.568500 & 1.726800 \\ \mathrm{H} & 2.622600 & 0.216000 & 0.577200\end{array}$

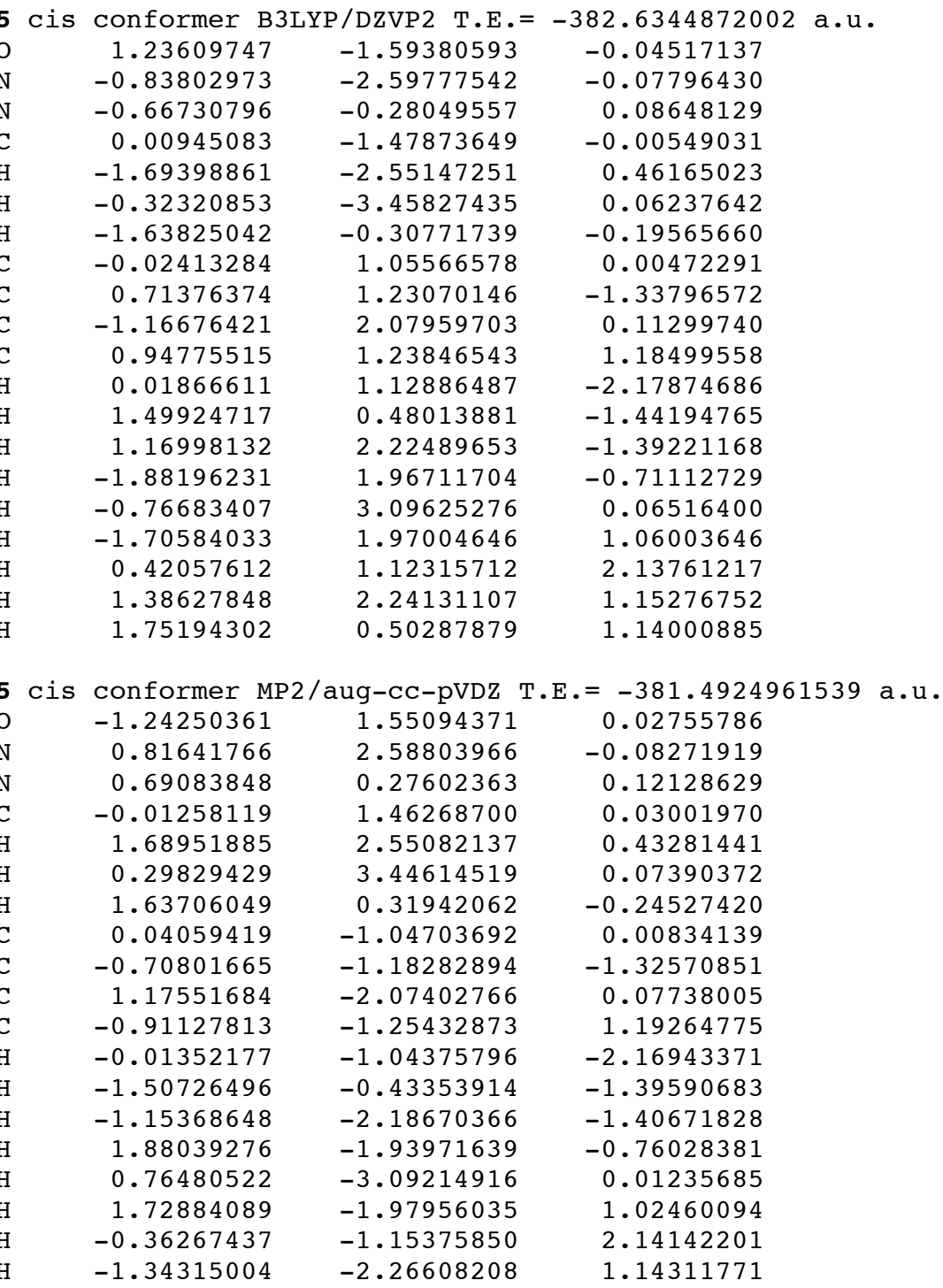


5 cis conformer MMFF94+

$\begin{array}{lrrr}\mathrm{O} & -1.079363 & -1.783315 & 0.236791 \\ \mathrm{~N} & -2.367641 & -0.093054 & -0.498408 \\ \mathrm{~N} & -0.159830 & 0.281460 & -0.266042 \\ \mathrm{C} & -1.170127 & -0.626999 & -0.140066 \\ \mathrm{H} & -2.524759 & 0.854367 & -0.186005 \\ \mathrm{H} & -3.136125 & -0.718986 & -0.288201 \\ \mathrm{H} & -0.357501 & 0.974650 & -0.973770 \\ \mathrm{C} & 1.261610 & -0.037499 & -0.060183 \\ \mathrm{C} & 1.745139 & -1.067340 & -1.092759 \\ \mathrm{C} & 2.066185 & 1.262253 & -0.244518 \\ \mathrm{C} & 1.517411 & -0.558796 & 1.363912 \\ \mathrm{H} & 1.556018 & -0.718154 & -2.114408 \\ \mathrm{H} & 1.234598 & -2.029233 & -0.977874 \\ \mathrm{H} & 2.820674 & -1.252402 & -0.993684 \\ \mathrm{H} & 1.925894 & 1.675187 & -1.250417 \\ \mathrm{H} & 3.139037 & 1.094059 & -0.098401 \\ \mathrm{H} & 1.744433 & 2.029775 & 0.469158 \\ \mathrm{H} & 1.130063 & 0.140435 & 2.113882 \\ \mathrm{H} & 2.589578 & -0.691011 & 1.549111 \\ \mathrm{H} & 1.038628 & -1.526868 & 1.540959\end{array}$

\begin{tabular}{|c|c|c|}
\hline eer & B3LYP & -382.63 \\
\hline-0.92799044 & -2.53208885 & 0.01929605 \\
\hline 1.21833936 & -1.71884456 & 0.03330277 \\
\hline-0.64353141 & -0.29419631 & -0.34737831 \\
\hline-0.16365960 & -1.56939084 & -0.09227781 \\
\hline 1.80105420 & -1.11600918 & -0.53088832 \\
\hline 1.48515123 & -2.69496775 & 0.00694637 \\
\hline-1.65575083 & -0.31916653 & -0.32022999 \\
\hline-0.06105506 & 1.02750746 & 0.01072813 \\
\hline 1.16050980 & 1.36682233 & -0.86943232 \\
\hline-1.17122050 & 2.05193417 & -0.28870034 \\
\hline 0.31565274 & 1.09078420 & 1.50511829 \\
\hline 0.94147227 & 1.18323249 & -1.92531434 \\
\hline 2.05273966 & 0.79978694 & -0.58722777 \\
\hline 1.41963194 & 2.42345545 & -0.75019589 \\
\hline-1.44628296 & 2.03279514 & -1.34826299 \\
\hline-0.83403650 & 3.06181774 & -0.03939185 \\
\hline-2.06782007 & 1.84382807 & 0.30595058 \\
\hline-0.56686252 & 0.92421212 & 2.13095989 \\
\hline 0.73132405 & 2.07331897 & 1.75340840 \\
\hline 1.05837530 & 0.32954213 & 1.75570223 \\
\hline ns conformer & MP2 / aug-cc-pVDZ & T.E. $=-381.4883290933 \mathrm{a} . \mathrm{u}$ \\
\hline-0.92850313 & -2.49569474 & 0.03054855 \\
\hline 1.22310885 & -1.67821892 & 0.03050772 \\
\hline-0.60921730 & -0.27742633 & -0.48002834 \\
\hline-0.15645068 & -1.54588252 & -0.12741131 \\
\hline 1.79503344 & -1.12760765 & -0.59851292 \\
\hline 1.48903996 & -2.65693696 & 0.06731575 \\
\hline-1.62709376 & -0.30125220 & -0.46735399 \\
\hline-0.06258579 & 1.01235747 & 0.01189874 \\
\hline 1.28441021 & 1.34783062 & -0.64685735 \\
\hline-1.08813936 & 2.06771008 & -0.42148885 \\
\hline 0.07390525 & 1.00918763 & 1.54130554 \\
\hline 1.23833027 & 1.16680291 & -1.73135746 \\
\hline 2.11417151 & 0.77381935 & -0.21071064 \\
\hline 1.51556943 & 2.41148424 & -0.48272214 \\
\hline-1.18248631 & 2.08699939 & -1.51808638 \\
\hline-0.77539123 & 3.06399616 & -0.07630751 \\
\hline-2.07745898 & 1.85188942 & 0.01345851 \\
\hline-0.90538614 & 0.83437927 & 2.01295871 \\
\hline
\end{tabular}




$\begin{array}{llll}\mathrm{H} & 0.46235562 & 1.97815541 & 1.89301688 \\ \mathrm{H} & 0.76542120 & 0.21822355 & 1.86534944\end{array}$

$\begin{array}{lrrr}5 & \text { trans conformer MMFF94+ } & \\ \mathrm{O} & -2.518298 & -1.293396 & 0.493464 \\ \mathrm{~N} & -1.984719 & 0.868659 & 0.339856 \\ \mathrm{~N} & -0.364611 & -0.771642 & 0.184998 \\ \mathrm{C} & -1.656455 & -0.432955 & 0.354804 \\ \mathrm{H} & -1.344123 & 1.521299 & 0.757326 \\ \mathrm{H} & -2.955091 & 1.029764 & 0.577726 \\ \mathrm{H} & -0.263232 & -1.779543 & 0.210757 \\ \mathrm{C} & 0.867541 & 0.010765 & -0.020936 \\ \mathrm{C} & 1.252665 & 0.776590 & 1.253929 \\ \mathrm{C} & 2.003010 & -0.985240 & -0.335632 \\ \mathrm{C} & 0.742240 & 0.972430 & -1.212746 \\ \mathrm{H} & 1.322964 & 0.098460 & 2.112360 \\ \mathrm{H} & 0.526277 & 1.551031 & 1.512973 \\ \mathrm{H} & 2.223531 & 1.271914 & 1.137750 \\ \mathrm{H} & 2.142629 & -1.698432 & 0.485401 \\ \mathrm{H} & 2.956394 & -0.469900 & -0.497763 \\ \mathrm{H} & 1.777660 & -1.567594 & -1.236877 \\ \mathrm{H} & 0.408660 & 0.442374 & -2.112233 \\ \mathrm{H} & 1.704402 & 1.446158 & -1.439467 \\ \mathrm{H} & 0.028519 & 1.779182 & -1.023429\end{array}$

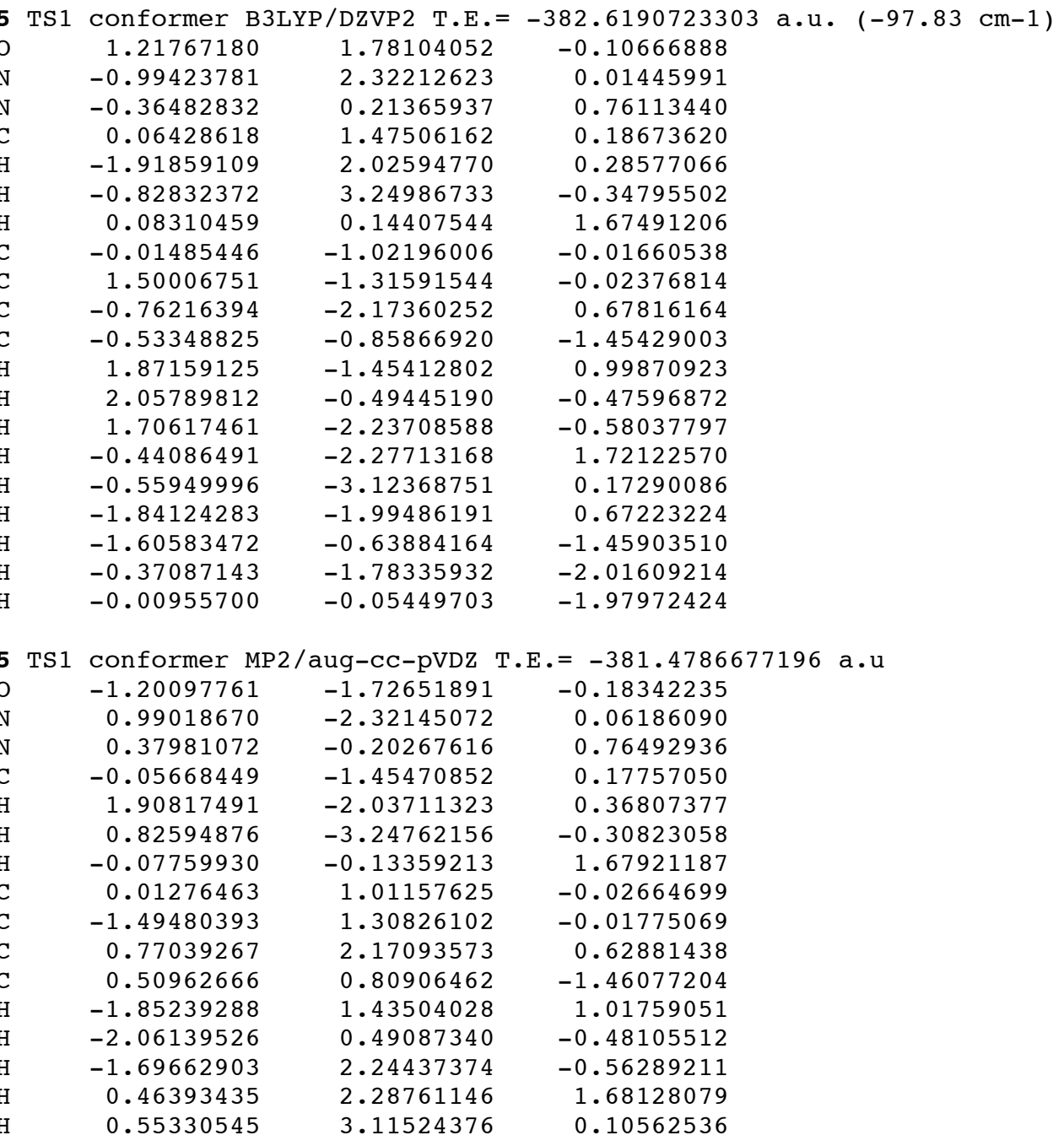




$\begin{array}{rrrr}\mathrm{H} & 1.85419237 & 1.98578072 & 0.60263392 \\ \mathrm{H} & 1.58487616 & 0.57376755 & -1.46655633 \\ \mathrm{H} & 0.35119636 & 1.73033262 & -2.04138361 \\ \mathrm{H} & -0.03696866 & -0.00465113 & -1.96121889\end{array}$

\begin{tabular}{lrrr} 
5 TS1 Conformer & \multicolumn{3}{l}{ MMFF94+ } \\
$\mathrm{O}$ & -1.561300 & 0.602600 & 1.435750 \\
$\mathrm{~N}$ & -2.268100 & -0.742900 & -0.238350 \\
$\mathrm{~N}$ & -0.106800 & -0.843100 & 0.398550 \\
$\mathrm{C}$ & -1.323000 & -0.249400 & 0.596450 \\
$\mathrm{H}$ & -1.937300 & -1.123700 & -1.112150 \\
$\mathrm{H}$ & -3.133800 & -0.222000 & -0.260650 \\
$\mathrm{H}$ & 0.110600 & -1.349100 & 1.248150 \\
$\mathrm{C}$ & 1.065400 & -0.029700 & 0.016050 \\
$\mathrm{C}$ & 1.480200 & 0.935800 & 1.138750 \\
$\mathrm{C}$ & 2.230200 & -0.996100 & -0.260150 \\
$\mathrm{C}$ & 0.777500 & 0.767500 & -1.267750 \\
$\mathrm{H}$ & 1.633600 & 0.400400 & 2.082650 \\
$\mathrm{H}$ & 0.722300 & 1.705700 & 1.315650 \\
$\mathrm{H}$ & 2.414100 & 1.452600 & 0.889750 \\
$\mathrm{H}$ & 2.476000 & -1.583800 & 0.632150 \\
$\mathrm{H}$ & 3.133800 & -0.457900 & -0.567350 \\
$\mathrm{H}$ & 1.973900 & -1.705700 & -1.055650 \\
$\mathrm{H}$ & 0.466400 & 0.103600 & -2.082650 \\
$\mathrm{H}$ & 1.665100 & 1.317000 & -1.600850 \\
$\mathrm{H}$ & -0.025100 & 1.498100 & -1.116750
\end{tabular}

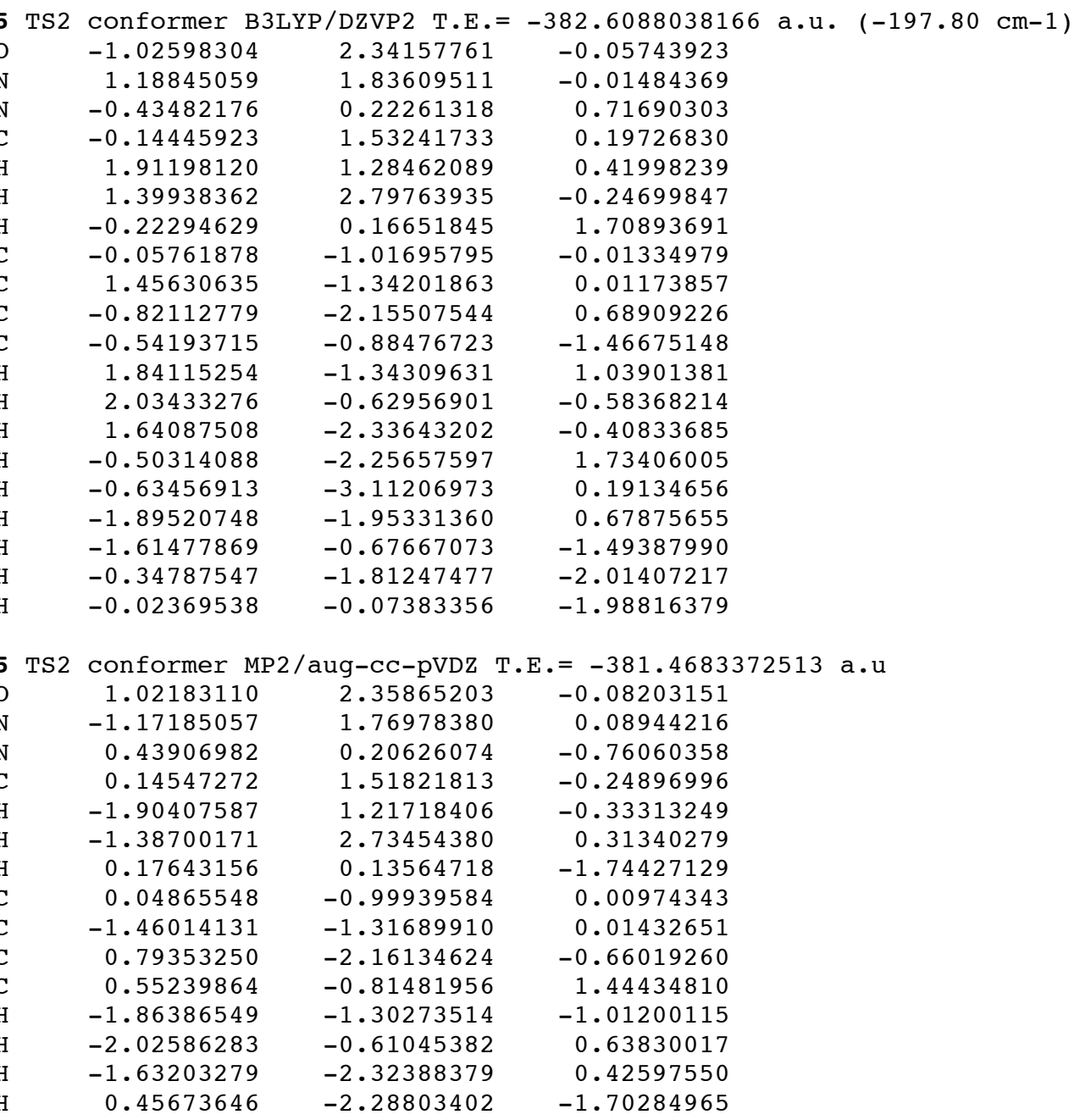




$\begin{array}{lrrr}\mathrm{H} & 0.60060683 & -3.10377954 & -0.12492023 \\ \mathrm{H} & 1.87429555 & -1.96192466 & -0.66663488 \\ \mathrm{H} & 1.63385021 & -0.61639943 & 1.44442093 \\ \mathrm{H} & 0.35428682 & -1.72529226 & 2.03041951 \\ \mathrm{H} & 0.04127613 & 0.02580861 & 1.93823565\end{array}$

5 TS2 conformer MMFF94+

$\begin{array}{rrrr}\mathrm{O} & -2.632950 & -0.704200 & -0.193800 \\ \mathrm{~N} & -1.813150 & 0.657000 & 1.389500 \\ \mathrm{~N} & -0.417950 & -0.806000 & 0.318800 \\ \mathrm{C} & -1.672250 & -0.294400 & 0.441200 \\ \mathrm{H} & -1.210250 & 0.612600 & 2.195700 \\ \mathrm{H} & -2.778450 & 0.896100 & 1.578400 \\ \mathrm{H} & -0.217050 & -1.474000 & 1.049300 \\ \mathrm{C} & 0.755550 & -0.031500 & -0.125800 \\ \mathrm{C} & 1.260550 & 0.927300 & 0.963500 \\ \mathrm{C} & 1.875850 & -1.036500 & -0.448900 \\ \mathrm{C} & 0.431250 & 0.762900 & -1.402200 \\ \mathrm{H} & 1.413850 & 0.400800 & 1.912300 \\ \mathrm{H} & 0.558050 & 1.746300 & 1.146600 \\ \mathrm{H} & 2.214150 & 1.384500 & 0.675000 \\ \mathrm{H} & 2.149050 & -1.621800 & 0.436900 \\ \mathrm{H} & 2.778450 & -0.529900 & -0.808300 \\ \mathrm{H} & 1.558150 & -1.746300 & -1.221800 \\ \mathrm{H} & 0.063550 & 0.102000 & -2.195700 \\ \mathrm{H} & 1.317650 & 1.283900 & -1.781400 \\ \mathrm{H} & -0.342350 & 1.517500 & -1.220700\end{array}$

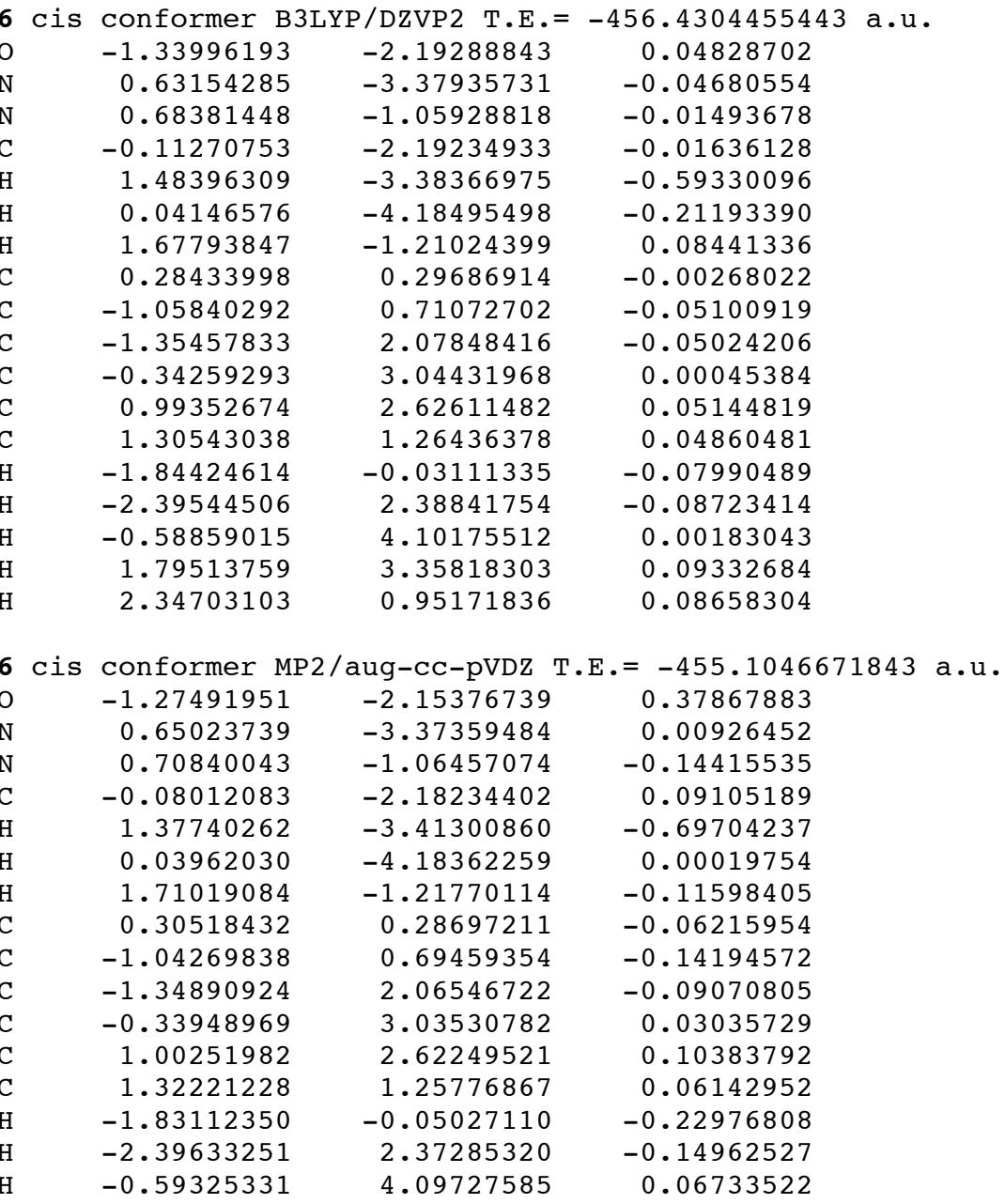




$\begin{array}{llll}\mathrm{H} & 1.80363129 & 3.35985670 & 0.19955697 \\ \mathrm{H} & 2.36863681 & 0.94069173 & 0.12317215\end{array}$

$\begin{array}{lrrr}6 & \text { cis } \text { conformer } & \text { MMF } 94+ & \\ \mathrm{O} & -2.366281 & 0.008117 & 0.806610 \\ \mathrm{~N} & -2.952202 & -1.395013 & -0.838854 \\ \mathrm{~N} & -0.744789 & -1.015594 & -0.527176 \\ \mathrm{C} & -2.014700 & -0.728360 & -0.102344 \\ \mathrm{H} & -2.790634 & -1.413048 & -1.835750 \\ \mathrm{H} & -3.891464 & -1.102721 & -0.593865 \\ \mathrm{H} & -0.668857 & -1.866089 & -1.065038 \\ \mathrm{C} & 0.451224 & -0.488433 & -0.003156 \\ \mathrm{C} & 0.510586 & 0.718406 & 0.696826 \\ \mathrm{C} & 1.734213 & 1.200940 & 1.170273 \\ \mathrm{C} & 2.906779 & 0.484779 & 0.939531 \\ \mathrm{C} & 2.857982 & -0.713794 & 0.231771 \\ \mathrm{C} & 1.635135 & -1.196685 & -0.241400 \\ \mathrm{H} & -0.383438 & 1.306710 & 0.883586 \\ \mathrm{H} & 1.766924 & 2.138630 & 1.719164 \\ \mathrm{H} & 3.856256 & 0.862086 & 1.310080 \\ \mathrm{H} & 3.771354 & -1.273522 & 0.048440 \\ \mathrm{H} & 1.622270 & -2.133892 & -0.790707\end{array}$

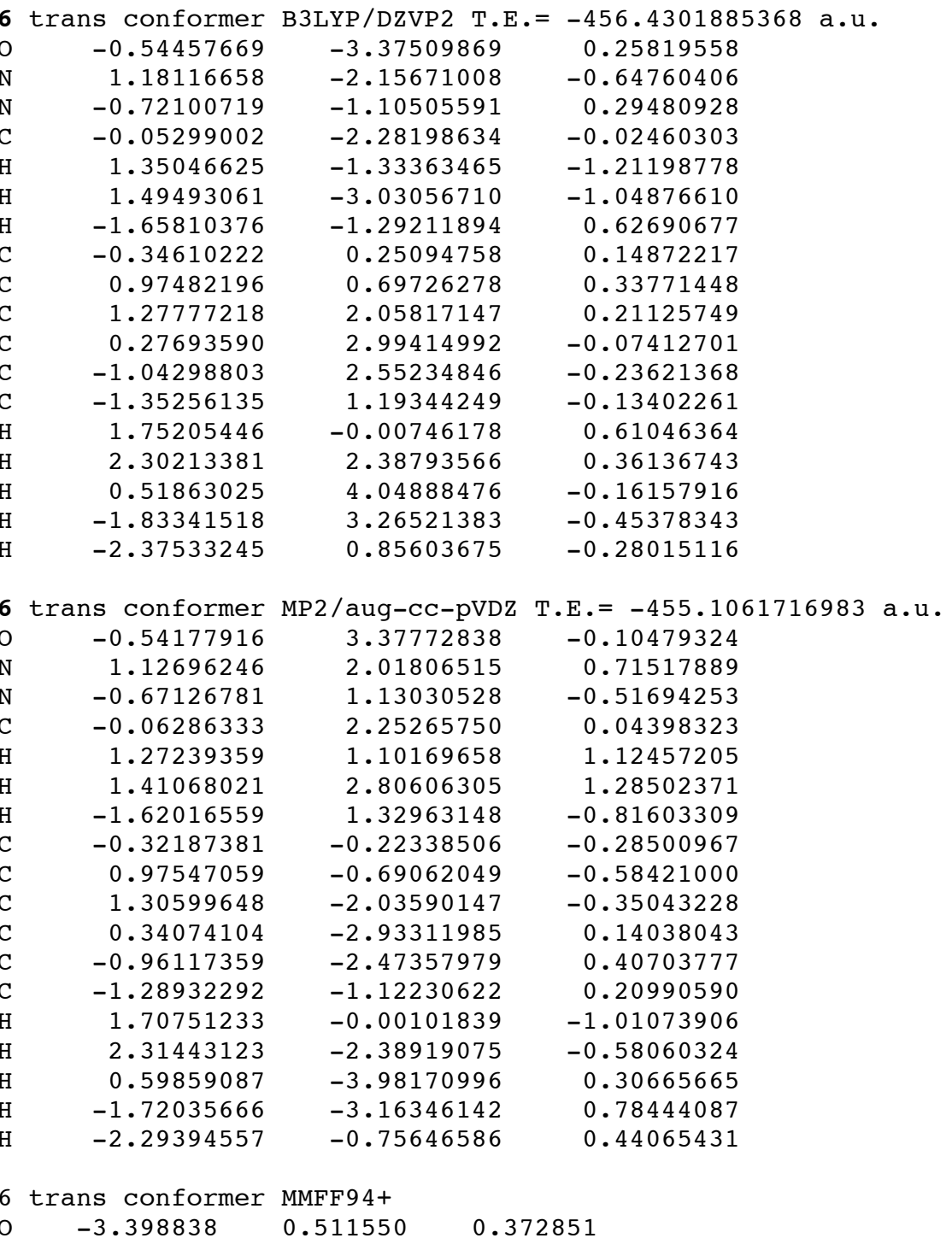




$\begin{array}{lrrr}\mathrm{N} & -2.126820 & -1.301315 & -0.005076 \\ \mathrm{~N} & -1.231173 & 0.821638 & -0.195854 \\ \mathrm{C} & -2.304078 & 0.036219 & 0.094443 \\ \mathrm{H} & -1.423904 & -1.615806 & -0.659063 \\ \mathrm{H} & -2.986025 & -1.834089 & 0.010225 \\ \mathrm{H} & -1.442168 & 1.807649 & -0.094681 \\ \mathrm{C} & 0.127606 & 0.478962 & -0.097793 \\ \mathrm{C} & 0.620683 & -0.316171 & 0.939270 \\ \mathrm{C} & 1.985254 & -0.602993 & 1.009887 \\ \mathrm{C} & 2.858145 & -0.086917 & 0.052487 \\ \mathrm{C} & 2.369077 & 0.721470 & -0.973192 \\ \mathrm{C} & 1.005057 & 1.009839 & -1.045033 \\ \mathrm{H} & -0.044123 & -0.708532 & 1.704682 \\ \mathrm{H} & 2.367545 & -1.223378 & 1.816561 \\ \mathrm{H} & 3.920820 & -0.309301 & 0.109968 \\ \mathrm{H} & 3.050348 & 1.129176 & -1.715665 \\ \mathrm{H} & 0.634023 & 1.641222 & -1.848194\end{array}$

\begin{tabular}{|c|c|c|c|c|}
\hline \multicolumn{5}{|c|}{50} \\
\hline 0.34037923 & \multicolumn{2}{|c|}{2.31991204} & 1.30660461 & \\
\hline 0.22934370 & \multicolumn{2}{|c|}{3.00444717} & -0.87303831 & \\
\hline-0.89881123 & \multicolumn{2}{|c|}{1.05761371} & -0.27809269 & \\
\hline-0.05425979 & \multicolumn{2}{|c|}{2.15989664} & 0.16072429 & \\
\hline-0.12180328 & \multicolumn{2}{|c|}{2.80642107} & -1.79729408 & \\
\hline 0.78711812 & \multicolumn{2}{|c|}{3.82739201} & -0.69567691 & \\
\hline-1.81012905 & \multicolumn{2}{|c|}{1.15155397} & 0.16228767 & \\
\hline-0.39775157 & \multicolumn{2}{|c|}{-0.26472625} & -0.11350291 & \\
\hline 0.97654120 & \multicolumn{2}{|c|}{-0.54482498} & -0.19444068 & \\
\hline 1.42739770 & \multicolumn{2}{|c|}{-1.86606371} & -0.11098086 & \\
\hline 0.52555629 & \multicolumn{2}{|c|}{-2.92496000} & 0.05283065 & \\
\hline-0.84418650 & -2.643 & 1199 & 0.13623730 & \\
\hline-1.30509754 & -1.325 & 4279 & 0.05383285 & \\
\hline 1.69280067 & 0.263 & 7288 & -0.30761282 & \\
\hline 2.49381968 & -2.066 & 5738 & -0.16944719 & \\
\hline 0.88294147 & -3.947 & 1430 & 0.11882754 & \\
\hline-1.55913704 & -3.450 & 3884 & 0.26860791 & \\
\hline-2.37093324 & -1.119 & 4328 & 0.11682669 & \\
\hline conformer $\mathrm{M}$ & IP 2 / aug-CC- & VDZ & $=-455.0917394602 \mathrm{a} \cdot \mathrm{u}$ & \\
\hline 0.23822574 & -2.213 & 2563 & -1.34654269 & \\
\hline 0.32845440 & -3.001 & 8630 & 0.80376620 & \\
\hline-0.88783038 & -1.065 & 5454 & 0.40437973 & \\
\hline-0.06316358 & -2.124 & 4790 & -0.16271487 & \\
\hline 0.04987869 & -2.848 & 9057 & 1.76145980 & \\
\hline 0.88839899 & -3.802 & 5482 & 0.54592525 & \\
\hline-1.83304476 & -1.163 & 4933 & 0.03071018 & \\
\hline-0.40146046 & 0.253 & 2251 & 0.17461629 & \\
\hline 0.98200704 & 0.519 & 1996 & 0.19647736 & \\
\hline 1.44541101 & 1.836 & 9999 & 0.05312701 & \\
\hline 0.54087770 & 2.901 & 5817 & -0.11018960 & \\
\hline-0.83896853 & 2.632 & 8051 & -0.13474695 & \\
\hline-1.30863661 & 1.316 & 4961 & 0.00300010 & \\
\hline 1.69488942 & -0.300 & 8925 & 0.31923864 & \\
\hline 2.52127753 & 2.029 & 9039 & 0.06733075 & \\
\hline 0.90632926 & 3.924 & 7152 & -0.22183866 & \\
\hline-1.55531765 & 3.447 & 6456 & -0.26777224 & \\
\hline-2.38382158 & 1.112 & 3830 & -0.01729976 & \\
\hline conformer $\mathrm{M}$ & IMFF 94+ & & & \\
\hline-2.613700 & -0.523050 & 1.0 & 7050 & \\
\hline-2.849100 & -0.074650 & -1.1 & 6250 & \\
\hline-0.930400 & -1.047650 & -0.4 & 1050 & \\
\hline-2.155100 & -0.541850 & -0.1 & 5450 & \\
\hline-2.290400 & 0.268050 & -1.9 & 4850 & \\
\hline-3.670900 & 0.465150 & -0.9 & 1150 & \\
\hline
\end{tabular}




$\begin{array}{rrrr}\mathrm{H} & -0.933800 & -2.027650 & -0.202050 \\ \mathrm{C} & 0.272500 & -0.478150 & 0.033050 \\ \mathrm{C} & 0.304100 & 0.647550 & 0.860650 \\ \mathrm{C} & 1.527300 & 1.156550 & 1.304750 \\ \mathrm{C} & 2.721200 & 0.548650 & 0.919450 \\ \mathrm{C} & 2.695000 & -0.569250 & 0.087950 \\ \mathrm{C} & 1.473700 & -1.080450 & -0.355750 \\ \mathrm{H} & -0.609700 & 1.139550 & 1.182950 \\ \mathrm{H} & 1.545700 & 2.027650 & 1.954850 \\ \mathrm{H} & 3.670900 & 0.947050 & 1.267150 \\ \mathrm{H} & 3.625100 & -1.041850 & -0.216950 \\ \mathrm{H} & 1.469500 & -1.946450 & -1.012650\end{array}$

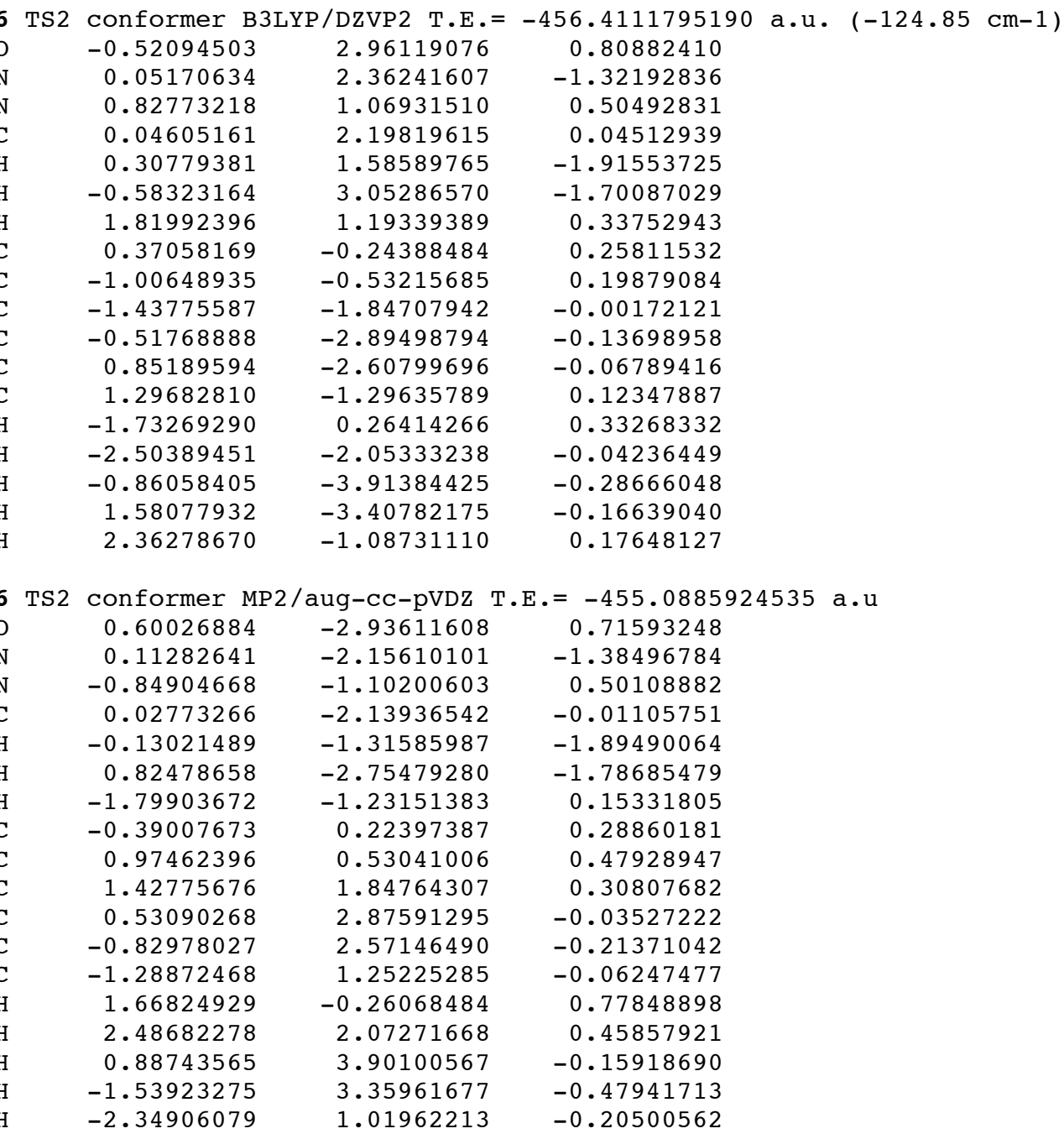

6 TS2 conformer MMFF94+

$\begin{array}{rrr}-3.150350 & 0.070700 & -1.011300 \\ -2.565750 & -1.473600 & 0.525500 \\ -1.091650 & -0.949200 & -1.135700 \\ -2.309950 & -0.694300 & -0.561200 \\ -1.780750 & -1.648800 & 1.137800 \\ -3.418550 & -1.209400 & 1.003800 \\ -1.025650 & -1.933800 & -1.362300 \\ 0.100150 & -0.466300 & -0.537100 \\ 0.119250 & 0.714500 & 0.212000 \\ 1.312250 & 1.161600 & 0.783500 \\ 2.489350 & 0.437700 & 0.599900\end{array}$




$\begin{array}{lrrr}\mathrm{C} & 2.477750 & -0.729000 & -0.163400 \\ \mathrm{C} & 1.286250 & -1.178300 & -0.736800 \\ \mathrm{H} & -0.782650 & 1.307900 & 0.343500 \\ \mathrm{H} & 1.323850 & 2.081800 & 1.362300 \\ \mathrm{H} & 3.418550 & 0.790500 & 1.040600 \\ \mathrm{H} & 3.399150 & -1.284600 & -0.318900 \\ \mathrm{H} & 1.300250 & -2.081800 & -1.340300\end{array}$


<smiles>CCCCCC[C@@H](C)N(C)C(N)=O</smiles>

Table 1S. Calculated and experimental geometric parameters for cis $\mathbf{3 a} .^{a}$

\begin{tabular}{cccccc}
\hline Feature & DFT & MP2 & MMFF94 & MMFF94+ & Expt. $^{\mathrm{b}}$ \\
\hline $\mathrm{C}_{\mathrm{a}}=\mathrm{O}$ & 1.231 & 1.233 & 1.220 & 1.221 & $1.236 \pm 0.012$ \\
$\mathrm{C}_{\mathrm{a}}-\mathrm{N}_{1}$ & 1.402 & 1.399 & 1.358 & 1.357 & $1.377 \pm 0.029$ \\
$\mathrm{C}_{\mathrm{a}}-\mathrm{N}_{2}$ & 1.384 & 1.385 & 1.364 & 1.363 & $1.335 \pm 0.012$ \\
$\mathrm{C}_{4}-\mathrm{N}_{2}$ & 1.464 & 1.464 & 1.448 & 1.448 & $1.453 \pm 0.012$ \\
$\mathrm{~N}_{2}-\mathrm{H}_{3}$ & 1.012 & 1.015 & 1.009 & 1.009 & $0.894 \pm 0.062$ \\
$\mathrm{C}_{4}-\mathrm{C}_{5}$ & 1.536 & 1.529 & 1.518 & 1.519 & $1.511 \pm 0.017$ \\
& & & & & \\
$\mathrm{~N}_{1}-\mathrm{C}_{\mathrm{a}}-\mathrm{O}$ & 122.3 & 122.7 & 121.8 & 121.8 & $120.3 \pm 1.6$ \\
$\mathrm{~N}_{1}-\mathrm{C}_{\mathrm{a}}-\mathrm{N}_{2}$ & 114.0 & 113.8 & 112.0 & 112.0 & $116.2 \pm 1.9$ \\
$\mathrm{C}_{\mathrm{a}}-\mathrm{N}_{2}-\mathrm{C}_{4}$ & 120.0 & 118.1 & 121.0 & 121.0 & $121.8 \pm 1.4$ \\
$\mathrm{C}_{\mathrm{a}}-\mathrm{N}_{2}-\mathrm{H}_{3}$ & 116.1 & 115.2 & 114.9 & 115.0 & $118.4 \pm 3.2$ \\
$\mathrm{~N}_{2}-\mathrm{C}_{4}-\mathrm{C}_{5}$ & 113.8 & 113.2 & 112.1 & 112.1 & $112.2 \pm 2.0$ \\
& & & & & \\
$O-\mathrm{C}_{\mathrm{a}}-\mathrm{N}_{2}-\mathrm{C}_{4}$ & 9.3 & 11.8 & 4.2 & 5.5 & $0 \pm 3.2(0-12)^{\mathrm{c}}$ \\
$\mathrm{O}-\mathrm{C}_{\mathrm{a}}-\mathrm{N}_{2}-\mathrm{H}_{3}$ & 158.1 & 155.3 & 154.7 & 156.1 & $30 \pm 5.6(150-180)$ \\
$\gamma \mathrm{N}_{1}$ & 341.5 & 340.9 & 341.3 & 341.0 & \\
$\gamma \mathrm{N}_{2}$ & 352.5 & 349.5 & 353.3 & 353.3 & \\
\hline
\end{tabular}

${ }^{a}$ Bond lengths in $\AA$, bond angles in deg. Pyramidalization of the nitrogen is expressed in terms of $\gamma$, the sum of the three bond angles subtended at nitrogen. ${ }^{b}$ Experimental data from X-ray diffraction data for urea derivatives bearing cis primary alkyl substituents. 'Dihedral angles range. 


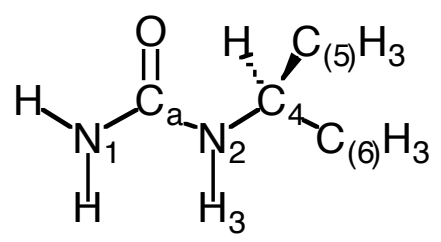

Table 2S. Calculated and experimental geometric parameters for cis $\mathbf{4 a}{ }^{a}$

\begin{tabular}{cccccc}
\hline Feature & DFT & MP2 & MMFF94 & MMFF94+ & Expt. $^{\mathrm{b}}$ \\
\hline $\mathrm{C}_{\mathrm{a}}=\mathrm{O}$ & 1.232 & 1.233 & 1.221 & 1.220 & $1.226 \pm 0.014$ \\
$\mathrm{C}_{\mathrm{a}}-\mathrm{N}_{1}$ & 1.403 & 1.399 & 1.357 & 1.358 & $1.392 \pm 0.029$ \\
$\mathrm{C}_{\mathrm{a}}-\mathrm{N}_{2}$ & 1.382 & 1.385 & 1.364 & 1.364 & $1.340 \pm 0.010$ \\
$\mathrm{C}_{4}-\mathrm{N}_{2}$ & 1.470 & 1.468 & 1.456 & 1.456 & $1.458 \pm 0.010$ \\
$\mathrm{~N}_{2}-\mathrm{H}_{3}$ & 1.013 & 1.016 & 1.010 & 1.010 & $0.970 \pm 0.100$ \\
$\mathrm{C}_{4}-\mathrm{C}_{5}$ & 1.538 & 1.531 & 1.528 & 1.528 & $1.506 \pm 0.016^{\mathrm{c}}$ \\
$\mathrm{C}_{4}-\mathrm{C}_{6}$ & 1.533 & 1.527 & 1.526 & 1.525 & \\
& & & & & \\
$\mathrm{~N}_{1}-\mathrm{C}_{\mathrm{a}}-\mathrm{O}$ & 122.1 & 122.7 & 121.8 & 121.8 & $121.8 \pm 2.3$ \\
$\mathrm{~N}_{1}-\mathrm{C}_{\mathrm{a}}-\mathrm{N}_{2}$ & 114.0 & 113.8 & 111.9 & 111.9 & $114.4 \pm 1.6$ \\
$\mathrm{C}_{\mathrm{a}}-\mathrm{N}_{2}-\mathrm{C}_{4}$ & 120.6 & 118.8 & 121.0 & 120.9 & $122.3 \pm 1.2$ \\
$\mathrm{C}_{\mathrm{a}}-\mathrm{N}_{2}-\mathrm{H}_{3}$ & 116.4 & 115.2 & 113.8 & 113.8 & $118.3 \pm 420$ \\
$\mathrm{~N}_{2}-\mathrm{C}_{4}-\mathrm{C}_{5}$ & 111.9 & 111.6 & 111.0 & 111.0 & $110.2 \pm 0.7^{\mathrm{c}}$ \\
$\mathrm{N}_{2}-\mathrm{C}_{4}-\mathrm{C}_{6}$ & 109.2 & 108.5 & 109.9 & 109.8 & \\
$\mathrm{C}_{5}-\mathrm{C}_{4}-\mathrm{C}_{6}$ & 112.2 & 112.0 & 110.7 & 110.7 & $111.7 \pm 1.5$ \\
& & & & & \\
$\mathrm{O}-\mathrm{C}_{\mathrm{a}}-\mathrm{N}_{2}-\mathrm{C}_{4}$ & 9.1 & 12.1 & 5.2 & 6.6 & $0 \pm 5.1(0-18)^{\mathrm{d}}$ \\
$\mathrm{O}_{-}-\mathrm{C}_{\mathrm{a}}-\mathrm{N}_{2}-\mathrm{H}_{3}$ & 158.7 & 155.3 & 152.4 & 153.7 & $180 \pm 6.8(152-180)$ \\
$\gamma \mathrm{N}_{1}$ & 341.8 & 341.1 & 341.2 & 340.7 & \\
$\gamma \mathrm{N}_{2}$ & 353.0 & 349.5 & 351.6 & 351.5 & \\
\hline
\end{tabular}

${ }^{a}$ Bond lengths in $\AA$, bond angles in deg. Pyramidalization of the nitrogen is expressed in terms of $\gamma$, the sum of the three bond angles subtended at nitrogen. ${ }^{b}$ Experimental data from X-ray diffraction data for cis isopropylurea derivatives. ${ }^{c}$ Average data for two carbon atoms of methyl groups. ${ }^{\mathrm{d}}$ Dihedral angles range. 
<smiles>CCC(C)(CC)N(C)C(=O)NC</smiles>

Table 3S. Calculated and experimental geometric parameters for $c i s \mathbf{5}^{\text {a }}$

\begin{tabular}{cccccc}
\hline Feature & DFT & MP2 & MMFF94 & MMFF94+ & Expt. $^{\mathrm{b}}$ \\
\hline $\mathrm{C}_{\mathrm{a}}=\mathrm{O}$ & 1.233 & 1.233 & 1.219 & 1.220 & $1.232 \pm 0.015$ \\
$\mathrm{C}_{\mathrm{a}}-\mathrm{N}_{1}$ & 1.406 & 1.402 & 1.358 & 1.359 & $1.388 \pm 0.033$ \\
$\mathrm{C}_{\mathrm{a}}-\mathrm{N}_{2}$ & 1.379 & 1.382 & 1.365 & 1.365 & $1.346 \pm 0.018$ \\
$\mathrm{C}_{4}-\mathrm{N}_{2}$ & 1.485 & 1.479 & 1.471 & 1.471 & $1.473 \pm 0.008$ \\
$\mathrm{~N}_{2}-\mathrm{H}_{3}$ & 1.011 & 1.016 & 1.010 & 1.010 & $0.850 \pm 0.070$ \\
$\mathrm{C}_{4}-\mathrm{C}_{5}$ & 1.542 & 1.536 & 1.537 & 1.536 & $1.519 \pm 0.009^{\mathrm{c}}$ \\
$\mathrm{C}_{4}-\mathrm{C}_{6}$ & 1.540 & 1.533 & 1.538 & 1.538 & \\
$\mathrm{C}_{4}-\mathrm{C}_{7}$ & 1.538 & 1.532 & 1.540 & 1.540 & \\
& & & & & \\
$\mathrm{~N}_{1}-\mathrm{C}_{\mathrm{a}}-\mathrm{O}$ & 121.6 & 122.1 & 121.4 & 121.3 & $120.5 \pm 2.9$ \\
$\mathrm{~N}_{1}-\mathrm{C}_{\mathrm{a}}-\mathrm{N}_{2}$ & 113.5 & 113.2 & 111.5 & 111.5 & $115.8 \pm 1.0$ \\
$\mathrm{C}_{\mathrm{a}}-\mathrm{N}_{2}-\mathrm{C}_{4}$ & 124.4 & 122.6 & 123.9 & 123.9 & $125.2 \pm 0.9$ \\
$\mathrm{C}_{\mathrm{a}} \mathrm{-}-\mathrm{N}_{2}-\mathrm{H}_{3}$ & 115.4 & 114.4 & 112.1 & 112.1 & $116.2 \pm 1.5$ \\
$\mathrm{~N}_{2}-\mathrm{C}_{4}-\mathrm{C}_{5}$ & 110.9 & 111.1 & 110.8 & 110.8 & $110.5 \pm 1.1^{\mathrm{d}}$ \\
$\mathrm{N}_{2}-\mathrm{C}_{4}-\mathrm{C}_{6}$ & 109.8 & 109.6 & 111.3 & 111.3 & \\
$\mathrm{~N}_{2}-\mathrm{C}_{4}-\mathrm{C}_{7}$ & 105.9 & 105.7 & 107.8 & 107.8 & $105.7 \pm 0.6$ \\
$\mathrm{C}_{5}-\mathrm{C}_{4}-\mathrm{C}_{6}$ & 110.6 & 110.9 & 110.0 & 110.0 & $110.1 \pm 0.4^{\mathrm{c}}$ \\
$\mathrm{C}_{5}-\mathrm{C}_{4}-\mathrm{C}_{7}$ & 110.0 & 109.9 & 108.7 & 108.7 & \\
$\mathrm{C}_{6}-\mathrm{C}_{4}-\mathrm{C}_{7}$ & 109.6 & 109.5 & 108.0 & 108.1 & \\
$\mathrm{O}_{2}-\mathrm{C}_{\mathrm{a}}-\mathrm{N}_{2}-\mathrm{C}_{4}$ & 6.9 & 10.1 & 4.1 & 5.2 & $0 \pm 3.3(0-19)^{\mathrm{e}}$ \\
$\mathrm{O}-\mathrm{C}_{\mathrm{a}}-\mathrm{N}_{2}-\mathrm{H}_{3}$ & 160.5 & 157.2 & 151.2 & 152.4 & $180 \pm 5.0(162-180)$ \\
$\gamma \mathrm{N}_{1}$ & 341.0 & 340.5 & 340.7 & 340.3 & \\
$\gamma \mathrm{N}_{2}$ & 355.0 & 351.9 & 351.8 & 351.8 & \\
\hline${ }^{2}-1.9$ & & & & \\
\hline
\end{tabular}

${ }^{a}$ Bond lengths in $\AA$, bond angles in deg. Pyramidalization of the nitrogen is expressed in terms of $\gamma$, the sum of the three bond angles subtended at nitrogen. ${ }^{b}$ Experimental data from x-ray diffraction data for $c i s$-tbutyllurea derivatives. ${ }^{\mathrm{c}}$ Average data for three carbon atoms of methyl groups. ${ }^{\mathrm{d} A v e r a g e}$ data for two carbon atoms of methyl groups. ${ }^{\mathrm{e}} \mathrm{Dihedral}$ angles range. 